\title{
THE SUMMABILITY OF THE DEVELOPMENTS IN BESSEL FUNCTIONS,
}

\section{WITH APPLICATIONS*}

BY

\author{
CHARLES N. MOORE
}

\section{Introduction.}

FeJfr has shown $\dagger$ that the Fourier development corresponding to a function $f(x)$, of period $2 \pi$, will be summable $\ddagger$ to the value

$$
\frac{1}{2}[f(x+0)+f(x-0)]
$$

at all points at which the function $f(x)$ is continuous or has a finite jump, if $f(x)$ is finite and integrable or even if $f(x)$ becomes infinite at a finite number of points, provided it remains integrable. He has further shown in the article referred to that the development is uniformly summable throughout any closed interval which does not include a point of discontinuity of the function. He has used these properties in connection with a general theorem about convergence factors to establish the fact that the formal results obtained in the discussion of many problems in Mathematical Physics which involve the development of an arbitrary function in a Fourier's series really furnish a solution of the problem, even when the development of the function is a divergent series.

FEJER's idea of investigating the nature of the divergence of a development of an arbitrary function in terms of normal functions and then applying general theorems about convergence factors to determine the behavior of the series when such factors are introduced, can be applied to many other developments that occur in Mathematical Physics. The present paper is devoted to a study, from this point of view, of the developments in terms of Bessel functions.

Although the formal work of obtaining the development of an arbitrary function in terms of Bessel functions goes back to FourIer, the rigorous discussion of the conditions under which the development is convergent has not made equal progress with that discussion in the case of the ordinary Fourier's series. This is doubtless due to the less elementary character of the functions involved

* Presented to the Society, September 11, 1908.

†Cf. Mathematische Annalen, vol. 58 (1903-04), p. 51.

$\ddagger$ The word summable will be used in this article in a somewhat special sense. Cf. the definition in $\& 2$. 
and to the fact that the formulæ in the case of the Bessel functions are considerably more cumbersome. As far as I am able to learn the first and almost the only satisfactory investigation of the convergence of the development to the value of the function developed is due to DiNI.* KNESER has discussed, $\dagger$ from the standpoint of integral equations, the convergence of the development in terms of $J_{0}$, and has stated that similar methods will apply to the developments in terms of $J_{\nu}$ where $\nu>0$. A number of other discussions of the convergence of the development have been published, but I do not know of any treatment, besides those mentioned, in which the reasoning is complete.

The discussion of the summability of the development, given in this paper, is analogous in its general outlines to the discussion of the convergence given by HARNACK. $\ddagger$ HARNACK's treatment is not adequate but his method is suggestive.

\section{§1. Distribution of the roots of an equation involving Bessel functions.}

We wish to discuss in this section the distribution of the positive roots of the equation

$$
l \lambda J_{\nu}^{\prime}(\lambda)+h J_{\nu}(\lambda)=0,
$$

where $J_{\nu}(\lambda)$ represents a Bessel function of the $\nu$ th order, $\nu$ is a constant positive or zero, and $h$ and $l$ are any constants not both zero.

We find it convenient to consider first the following trigonometric equation

$$
\cos (\lambda-a)+\frac{k}{\lambda} \sin (\lambda-a)=0 \quad(k, a \text { constants })
$$

whose roots will be found to approximate to the roots of equation (1) for large values of $\lambda$.

Lemma 1. If we represent by $\lambda_{1}^{\prime}, \lambda_{2}^{\prime}, \lambda_{3}^{\prime}, \ldots$ the successive positive roots of equation (2), we have

$$
\left|\lambda_{m+1}^{\prime}-\lambda_{m}^{\prime}-\pi\right|<\frac{K_{1}}{\lambda_{m}^{\prime 2}} \quad(m=1,2,3, \cdots)
$$

where $K_{1}$ is a positive constant.

This lemma is obviously true if $k=0$. Let us then first consider the case in which $k>0$, and let $\lambda_{0}$ be any positive value of $\lambda$ such that

$$
\begin{gathered}
\cos \left(\lambda_{0}-a\right)=0, \quad \sin \left(\lambda_{0}-a\right)=-1, \\
\lambda_{0}>k .
\end{gathered}
$$

* Serie di Fourier (Pisa, 1880), pp. 246-269.

† Mathematisohe Annalen, vol. 63 (1906-07), p. 505.

$\ddagger$ Mathematische Annalen, vol. 35 (1890), p. 11. 
Since, in view of $(4), \cos (\lambda-a)$ steadily increases from 0 to $\frac{1}{2} \sqrt{3}$ and $\sin (\lambda-a)$ steadily increases from -1 to $-\frac{1}{2}$ while $\lambda$ increases from $\lambda_{0}$ to $\lambda_{0}+\frac{1}{3} \pi$, it follows that the expression on the left hand side of equation (2), which is negative for $\lambda=\lambda_{0}$ and, in view of (5), is positive for $\lambda=\lambda_{0}+\frac{1}{3} \pi$, must vanish at one and only one point in the interval $\lambda_{0} \leqq \lambda \leqq \lambda_{0}+\frac{1}{3} \pi$. Moreover, this expression is always positive in the interval $\lambda_{0}+\frac{1}{3} \pi \leqq \lambda \leqq \lambda_{0}+\pi$, and hence equation (2) has one and only one root in the interval $\lambda_{0} \leqq \lambda \leqq \lambda_{0}+\pi$.

If we denote this root by $\lambda_{m}^{\prime}$, we have

$$
\begin{aligned}
\cos \left(\lambda_{m}^{\prime}+\pi-a\right)+\frac{k}{\lambda_{m}^{\prime}+\pi} & \sin \left(\lambda_{m}^{\prime}+\pi-a\right) \\
= & \frac{k}{\lambda_{m}^{\prime}} \sin \left(\lambda_{m}^{\prime}-a\right)+\frac{k}{\lambda_{m}^{\prime}+\pi} \sin \left(\lambda_{m}^{\prime}+\pi-a\right) \\
= & \frac{\pi k \sin \left(\lambda_{m}^{\prime}-a\right)}{\lambda_{m}^{\prime}\left(\lambda_{m}^{\prime}+\pi\right)}<0 .
\end{aligned}
$$

Since $\cos (\lambda-a)+(k / \lambda) \sin (\lambda-a)$ is positive for $\lambda=\lambda_{0}+\pi$, it follows from (6) that $\lambda_{m+1}^{\prime}$ lies in the interval $\lambda_{0}+\pi<\lambda<\lambda_{m}^{\prime}+\pi$. Moreover we have from (6)

$$
\begin{aligned}
& \frac{\pi k \sin \left(\lambda_{m}^{\prime}+\pi-a\right)}{\lambda_{m}^{\prime}\left(\lambda_{m}^{\prime}+\pi\right)} \\
& \quad=\cos \left(\lambda_{m+1}^{\prime}-a\right)+\frac{k}{\lambda_{m+1}^{\prime}} \sin \left(\lambda_{m+1}^{\prime}-a\right)-\cos \left(\lambda_{m}^{\prime}+\pi-a\right)-\frac{k}{\lambda_{m}^{\prime}+\pi} \sin \left(\lambda_{m}^{\prime}+\pi-a\right)
\end{aligned}
$$

$$
\begin{aligned}
&>\cos \left(\lambda_{m+1}^{\prime}-a\right)-\cos \left(\lambda_{m}^{\prime}+\pi-a\right)+\frac{k}{\lambda_{m+1}^{\prime}} \sin \left(\lambda_{m}^{\prime}+\pi-a\right) \\
&-\frac{k}{\lambda_{m}^{\prime}+\pi} \sin \left(\lambda_{m}^{\prime}+\pi-a\right) \\
&=\cos \left(\lambda_{m+1}^{\prime}-a\right)-\cos \left(\lambda_{m}^{\prime}+\pi-a\right)+k \sin \left(\lambda_{m}^{\prime}+\pi-a\right)\left[\frac{\lambda_{m}^{\prime}+\pi-\lambda_{m+1}^{\prime}}{\left(\lambda_{m}^{\prime}+\pi\right) \lambda_{m+1}^{\prime}}\right] .
\end{aligned}
$$

But since from the Law of the Mean

$$
\cos \left(\lambda_{m+1}^{\prime}-a\right)-\cos \left(\lambda_{m}^{\prime}+\pi-a\right)=-\sin \xi \cdot\left[\lambda_{m+1}^{\prime}-\lambda_{m}^{\prime}-\pi\right]
$$

we have from (7)

$$
\left(\lambda_{m+1}^{\prime}-a<\xi<\lambda_{m}^{\prime}+\pi-a\right)
$$

$$
-\left[\lambda_{m+1}^{\prime}-\lambda_{m}^{\prime}-\pi\right]<\frac{k \sin \left(\lambda_{m}^{\prime}+\pi-a\right)}{\sin \xi}\left[\frac{\pi}{\left(\lambda_{m}^{\prime}+\pi\right) \lambda_{m}^{\prime}}-\frac{\lambda_{m}^{\prime}+\pi-\lambda_{m+1}^{\prime}}{\left(\lambda_{m}^{\prime}+\pi\right) \lambda_{m+1}^{\prime}}\right],
$$

and since, moreover,

$$
\sin \xi>\sin \left(\lambda_{m}^{\prime}+\pi-a\right)=-\sin \left(\lambda_{m}^{\prime}-a\right)>-\sin \left(\lambda_{0}+\frac{\pi}{3}-a\right)=\frac{1}{2}
$$

and $\lambda_{m+1}^{\prime}-\lambda_{m}^{\prime}-\pi$ is negative, we have finally 


$$
\begin{array}{r}
\left|\lambda_{m+1}^{\prime}-\lambda_{m}^{\prime}-\pi\right|<2 k\left[\frac{\pi}{\left(\lambda_{m}^{\prime}+\pi\right) \lambda_{m}^{\prime}}+\frac{\lambda_{m}^{\prime}+\pi-\lambda_{m+1}^{\prime}}{\left(\lambda_{m}^{\prime}+\pi\right) \lambda_{m+1}^{\prime}}\right] \\
<2 k\left[\frac{\pi}{\lambda_{m}^{\prime 2}}+\frac{\pi}{\lambda_{m}^{\prime 2}}\right]=\frac{4 \pi k}{\lambda_{m}^{\prime 2}}
\end{array}
$$

The inequality (8) has been derived under the assumption that $\lambda_{0}$, the root of $\cos (\lambda-a)=0$ that immediately precedes $\lambda_{m}^{\prime}$, is such that $\sin \left(\lambda_{0}-a\right)=-1$ and the inequality (5) is satisfied. The first restriction was made for definiteness and the proof of formula (8) can be carried through in a similar manner if (4) is replaced by

$$
\cos \left(\lambda_{0}-a\right)=0, \quad \sin \left(\lambda_{0}-a\right)=1 .
$$

Moreover, since there are only a finite number of positive roots of equation (2) lying in the interval $0<\lambda<\bar{\lambda}_{0}$ where $\bar{\lambda}_{0}$ is the least value of $\lambda_{0}$ satisfying (4) and (5), or $\left(4^{\prime}\right)$ and (5), we may choose $K_{1}>4 \pi k$ and such that the inequality (3) is satisfied for all positive roots of (2) that are less than $\bar{\lambda}_{0}$. Hence, in view of $(8)$, we have

$$
\left|\lambda_{m+1}^{\prime}-\lambda_{m}^{\prime}-\pi\right|<\frac{K_{1}}{\lambda_{m}^{\prime 2}} \quad(m=1,2,3, \cdots)
$$

and the lemma is proved for the case where $k>0$.

The proof for the case where $k<0$ is quite analogous, the principal difference being that the successive roots of (2) come before instead of after the corresponding roots of $\cos (\lambda-a)=0$ to which they approximate.

We will consider next the equation

$$
\cos (\lambda-a)+\frac{k}{\lambda} \sin (\lambda-a)+\epsilon(\lambda)=0
$$

where $k$ and $a$ are constants and $\epsilon(\lambda)$ is a continuous function of $\lambda$ satisfying the inequality

$$
|\epsilon(\lambda)|<\frac{M}{\lambda^{2}} \quad \text { (M a positive constant) }
$$

Lемма 2. Corresponding to each root $\lambda_{m}^{\prime}$ of equation (2) there is a root $\lambda_{n}$ of equation (9) such that

where $K_{2}$ is a positive constant.

$$
\left|\lambda_{n}-\lambda_{m}^{\prime}\right|<\frac{K_{2}}{\lambda_{m}^{\prime 2}}
$$

Let us first consider the case where $k>0$; and let $\lambda_{0}$ be any positive value of $\lambda$ greater than $\frac{3}{4} \pi$ and such that

$$
\begin{array}{cc}
\cos \left(\lambda_{0}-a\right)=0, & \sin \left(\lambda_{0}-a\right)=-1 ; \\
\frac{k}{\lambda_{0}-\frac{1}{6} \pi}<\frac{1}{8}, & \frac{M}{\left(\lambda_{0}-\frac{1}{6} \pi\right)^{2}}<\frac{\pi}{48} .
\end{array}
$$


Then equation (2) will have one and only one root in the interval $\lambda_{0}<\lambda<\lambda_{0}+\frac{1}{12} \pi$. Denote that root by $\lambda_{m}^{\prime}$. For any positive increment $\Delta \lambda$ we have

$$
\begin{aligned}
& \cos \left(\lambda_{m}^{\prime}+\Delta \lambda-a\right)+\frac{k}{\lambda_{m}^{\prime}+\Delta \lambda} \sin \left(\lambda_{m}^{\prime}+\Delta \lambda-a\right) \\
& =\cos \left(\lambda_{m}^{\prime}+\Delta \lambda-a\right)+\frac{k}{\lambda_{m}^{\prime}+\Delta \lambda} \sin \left(\lambda_{m}^{\prime}+\Delta \lambda-a\right)-\cos \left(\lambda_{m}^{\prime}-a\right)-\frac{k}{\lambda_{m}^{\prime}} \sin \left(\lambda_{m}^{\prime}-a\right)
\end{aligned}
$$

$$
\begin{array}{r}
=\cos \left(\lambda_{m}^{\prime}+\Delta \lambda-a\right)-\cos \left(\lambda_{m}^{\prime}-a\right)-\frac{k \Delta \lambda}{\lambda_{m}^{\prime}\left(\lambda_{m}^{\prime}+\Delta \lambda\right)} \sin \left(\lambda_{m}^{\prime}+\Delta \lambda-a\right) \\
+\frac{k}{\lambda_{m}^{\prime}}\left[\sin \left(\lambda_{m}^{\prime}+\Delta \lambda-a\right)-\sin \left(\lambda_{m}^{\prime}-a\right)\right] .
\end{array}
$$

But

$$
\cos \left(\lambda_{m}^{\prime}+\Delta \lambda-a\right)-\cos \left(\lambda_{m}^{\prime}-a\right)=-\Delta \lambda \sin \left(\lambda_{m}^{\prime}+\theta \Delta \lambda-a\right) \quad(0<\theta<1),
$$

or if we take $\Delta \lambda<\frac{1}{4} \pi$ so that

$$
-1<\sin \left(\lambda_{m}^{\prime}+\theta \Delta \lambda-a\right)<\sin \left(\lambda_{0}+\frac{\pi}{3}-a\right)=-\frac{1}{2},
$$

$$
\cos \left(\lambda_{m}^{\prime}+\Delta \lambda-a\right)-\cos \left(\lambda_{m}^{\prime}-a\right)>\frac{\Delta \lambda}{2} \text {. }
$$

Moreover, in view of (13),

and since

$$
\left|\frac{k \Delta \lambda}{\lambda_{m}^{\prime}\left(\lambda_{m}^{\prime}+\Delta \lambda\right)} \sin \left(\lambda_{m}^{\prime}+\Delta \lambda-a\right)\right|<\frac{k}{\lambda_{m}^{\prime}} \Delta \lambda<\frac{\Delta \lambda}{8},
$$

$$
\sin \left(\lambda_{m}^{\prime}+\Delta \lambda-a\right)-\sin \left(\lambda_{m}^{\prime}-a\right)=\Delta \lambda \cos \left(\lambda_{m}^{\prime}+\theta_{1} \Delta \lambda-a\right) \quad\left(0<\theta_{1}<1\right),
$$

we have from (13)

$$
\frac{k}{\lambda_{m}^{\prime}} \cdot\left|\sin \left(\lambda_{m}^{\prime}+\Delta \lambda-a\right)-\sin \left(\lambda_{m}^{\prime}-a\right)\right|<\frac{\Delta \lambda}{8} .
$$

Combining (14) with (14a), (14b), and (14c), we obtain

$$
\cos \left(\lambda_{m}^{\prime}+\Delta \lambda-a\right)+\frac{k}{\lambda_{m}^{\prime}+\Delta \lambda} \sin \left(\lambda_{m}^{\prime}+\Delta \lambda-a\right)>\frac{\Delta \lambda}{4} \quad\left(0<\Delta i<\frac{\tau}{4}\right)
$$

If now we choose $\Delta \lambda$ so that

$$
\Delta \lambda=\frac{4 M}{\left(\lambda_{0}-\frac{1}{6} \pi\right)^{2}}<\frac{\pi}{12},
$$

we have, since $\lambda_{m}^{\prime}+\Delta \lambda>\lambda_{0}-\frac{1}{8} \pi$,

$$
\frac{\Delta \lambda}{4}=\frac{M}{\left(\lambda_{0}-\frac{1}{6} \pi\right)^{2}}>\frac{M}{\left(\lambda_{m}^{\prime}+\Delta \lambda\right)^{2}},
$$


and hence, in view of $(10)$ and (15),

$$
\cos \left(\lambda_{m}^{\prime}+\Delta \lambda-a\right)+\frac{k}{\lambda_{m}^{\prime}+\Delta \lambda} \sin \left(\lambda_{m}^{\prime}+\Delta \lambda-a\right)+\epsilon\left(\lambda_{m}^{\prime}+\Delta \lambda\right)>0
$$

In a precisely similar manner it may be shown that

$$
\cos \left(\lambda_{m}^{\prime}-\Delta \lambda-a\right)+\frac{k}{\lambda_{m}^{\prime}-\Delta \lambda} \sin \left(\lambda_{m}^{\prime}-\Delta \lambda-a\right)+\epsilon\left(\lambda_{m}^{\prime}-\Delta \lambda\right)<0
$$

It follows from (17) and (18) that equation (9) has at least one root in the interval

$$
\lambda_{m}^{\prime}-\Delta \lambda<\lambda<\lambda_{m}^{\prime}+\Delta \lambda
$$

If we represent such a root by $\lambda_{n}$, we have from (16)

$$
\begin{aligned}
\left|\lambda_{n}-\lambda_{m}^{\prime}\right|<\frac{4 M}{\left(\lambda_{0}-\frac{1}{6} \pi\right)^{2}}<\frac{4 M}{\left(\lambda_{m}^{\prime}-\frac{1}{4} \pi\right)^{2}} & =\frac{4 M}{\lambda_{m}^{\prime 2}} \cdot \frac{\lambda_{m}^{\prime 2}}{\left(\lambda_{m}^{\prime}-\frac{1}{4} \pi\right)^{2}} \\
& =\frac{4 M}{\lambda_{m}^{\prime 2}} \cdot \frac{1}{\left(1-\frac{\pi}{4 \lambda_{m}^{\prime}}\right)^{2}}<\frac{9 M}{\lambda_{m}^{\prime 2}} .
\end{aligned}
$$

The inequality (19) has been derived under the assumption that $\lambda_{0}$, the root of $\cos (\lambda-a)=0$ that immediately precedes $\lambda_{m}^{\prime}$, is such that $\sin \left(\lambda_{0}-a\right)=-1$ and the inequalities (13) are satisfied. The first restriction was made for definiteness and the proof can be carried through in a similar manner if (12) is replaced by

$$
\cos \left(\lambda_{0}-a\right)=0, \quad \sin \left(\lambda_{0}-a\right)=1 .
$$

Moreover, since there are only a finite number of roots of equation (2) lying in the interval $0<\lambda<\bar{\lambda}_{0}$ where $\bar{\lambda}_{0}$ is the least value of $\lambda_{0}$ satisfying (12) and (13), or $\left(12^{\prime}\right)$ and (13), we may choose $K_{2}$ greater than $9 M$ and such that the inequality (11) is satisfied for all the positive roots of (2) that are less than $\bar{\lambda}_{0}$, provided we choose as the $\lambda_{n}$ corresponding to each $\lambda_{m}^{\prime}$ that root of (9) which lies nearest to $\lambda_{m}^{\prime}$. Hence we have, in view of (19),

$$
\left|\lambda_{n}-\lambda_{m}^{\prime}\right|<\frac{K_{2}}{\lambda_{m}^{\prime 2}} \quad(m=1,2,3, \cdots),
$$

and the lemma is proved for the case of $k>0$. The proof for the case of $k<0$ is quite analogous, and for $k=0$ the proof is simpler.

We will now show the relationship between equation (9) and equation (1) by proving the following lemma:

Lемma 3. The positive roots of any equation of the form (1) are the same as the positive roots of a corresponding equation of the form (9). 
We obtain easily from the asymptotic expansion of $J_{\nu}(\lambda)^{*}$

(20) $J_{\nu}(\lambda)=\sqrt{\frac{2}{\pi \lambda}}\left[\cos \left(\lambda-\frac{2 \nu+1}{4} \pi\right)+\frac{1-4 \nu^{2}}{8 \lambda} \sin \left(\lambda-\frac{2 \nu+1}{4} \pi\right)+\epsilon_{\nu}(\lambda)\right]$

where

$$
\left|\epsilon_{\nu}(\lambda)\right|<\frac{M_{1}}{\lambda^{2}}
$$

$M_{1}$ being a positive constant.

We have for the derivative of $J_{\nu}(\lambda) \dagger$

$$
J_{\nu}^{\prime}(\lambda)=\frac{\nu}{\lambda} J_{\nu}(\lambda)-J_{\nu+1}(\lambda)
$$

and on substituting this value in equation (1) we get

$$
(\nu l+h) J_{\nu}(\lambda)-l \lambda J_{\nu+1}(\lambda)=0 .
$$

This equation may be thrown into another form by replacing $J_{\nu+1}(\lambda)$ by the value obtained by changing $\nu$ into $\nu+1$ in (20) and also replacing $J_{\nu}(\lambda)$ by

$$
-\sqrt{\frac{2}{\pi \lambda}}\left[\sin \left(\lambda-\frac{2 \nu+3}{4} \pi\right)+\bar{\epsilon}_{\nu}(\lambda)\right] \quad\left(\left|\bar{\varepsilon}_{\nu}(\lambda)\right|<\frac{M_{2}}{\lambda}\right),
$$

a value readily obtained from (20). We thus reduce (21) to the form

where

$$
\begin{aligned}
& -l \sqrt{\frac{2 \lambda}{\pi}}\left[\cos \left(\lambda-\frac{2 \nu+3}{4} \pi\right)\right. \\
& \left.\quad+\frac{8(\nu l+h)-l\left(4 \nu^{2}+8 \nu+3\right)}{8 l \lambda} \sin \left(\lambda-\frac{2 \nu+3}{4} \pi\right)+\epsilon(\lambda)\right]=0
\end{aligned}
$$

$M$ being a positive constant.

$$
|\epsilon(\lambda)|<\frac{M}{\lambda^{2}}
$$

Since the asymptotic expansions hold for all positive values of the argument, it follows that equation (22) is equivalent to equation (21) for all positive values of $\lambda$, and hence the positive roots of any equation of the form (1) are the same as the positive roots of an equation of the form (9). $\ddagger$

We are now ready to prove the following theorem:

TheOREM I. If we represent by $\lambda_{1}, \lambda_{2}, \lambda_{3}, \ldots$ the successive positive roots of equation (1), we have

$$
\lambda_{n}>C n, \quad\left|\lambda_{n+1}-\lambda_{n}-\pi\right|<\frac{K}{n^{2}}
$$

where $C$ and $K$ are positive constants.

* Cf. Lipschitz, Crelle's Jou rnal, vol. 56 (1859), pp. 193-196.

†Cf. Gray and Mathews, Treatise on Bessel Functions, p. 13.

$\ddagger$ There would seem to be an exception for the case $l=0$. However, in that case (21) reduces by the substitution of (20) to equation (26) given below which is of the form of equation (9). 
We prove this theorem first in the special case $l=0$, in which case (1) reduces to

$$
J_{\nu}(\lambda)=0 .
$$

STURM has shown * that for this case

$$
\lim _{n=\infty}\left(\lambda_{n+1}-\lambda_{n}\right)=\pi \text {. }
$$

From this fact the first inequality (23) follows at once.

In view of (20), equation (24) may be written for positive values of $\lambda$

(26) $\cos \left(\lambda-\frac{2 \nu+1}{4} \pi\right)+\frac{1-4 \nu^{2}}{8 \lambda} \sin \left(\lambda-\frac{2 \nu+1}{4} \pi\right)+\epsilon_{\nu}(\lambda)=0 \quad\left(\left|\varepsilon_{\nu}(\lambda)\right|<\frac{M_{1}}{\lambda^{2}}\right)$.

Hence, however small the positive quantity $\delta$, all the large roots of (24) lie in the intervals

$$
\left|\frac{2 \nu+1}{4} \pi+\frac{\pi}{2}+k \pi-\lambda\right|<\delta \quad(k=1,2, \cdots) .
$$

Since the distance between two successive roots of (24) approaches $\pi$ as its limit, it is clear that one and only one of these roots lies in each of the more distant of the intervals (27).

Precisely the same reasoning applies to the roots of the equation

$$
\cos \left(\lambda-\frac{2 \nu+1}{4}\right) \pi+\frac{1-4 \nu^{2}}{8 \lambda} \sin \left(\lambda-\frac{2 \nu+1}{4} \pi\right)=0,
$$

since the distance between two successive roots here also approaches $\pi$ as its limit, as we see from Lemma 1.

Hence all the large roots of $(26)$ coincide very nearly with the large roots of (28) and vice versa.

Let us now denote by $\lambda_{n}^{\prime}$ that positive root of (28) which nearly coincides with the large positive root $\lambda_{n}$ of $(26) \cdot \dagger$ Consequently $\lambda_{n}^{\prime}>C^{\prime} n$. By this inequality and Lemmas 1 and 2 we have for all large values of $n$

$$
\left|\lambda_{n}-\lambda_{n}^{\prime}\right|<\frac{K_{2}}{\lambda_{n}^{\prime 2}}<\frac{K_{2}^{\prime}}{n^{2}}, \quad\left|\lambda_{n+1}^{\prime}-\lambda_{n}^{\prime}-\pi\right|<\frac{K_{1}}{\lambda_{n}^{\prime 2}}<\frac{K_{1}^{\prime}}{n^{2}}
$$

For these large values of $n$ we therefore have

$$
\begin{aligned}
\left|\lambda_{n+1}-\lambda_{n}-\pi\right| & =\left|\left(\lambda_{n+1}-\lambda_{n+1}^{\prime}\right)-\left(\lambda_{n}-\lambda_{n}^{\prime}\right)+\left(\lambda_{n+1}^{\prime}-\lambda_{n}^{\prime}-\pi\right)\right| \\
& \leqq\left|\lambda_{n+1}-\lambda_{n+1}\right|+\left|\lambda_{n}-\lambda_{n}^{\prime}\right|+\left|\lambda_{n+1}^{\prime}-\lambda_{n}^{\prime}-\pi\right|<\frac{2 K_{2}^{\prime}+K_{1}^{\prime}}{n^{2}} .
\end{aligned}
$$

*Liouville's Journal, vol. 1 (1836), pp. 174-175.

$\dagger$ Notice that $\lambda_{n}^{\prime}$ is not necessarily the $n$th positive root of (28). However, $\lambda_{n}^{\prime}$ and $\lambda_{n+1}^{\prime}$ are successive positive roots of (28), and this is all that is essential. 
If then we take $K>2 K_{2}^{\prime}+K_{1}^{\prime}$, we see that the second inequality (23) is established for all large values of $n$. It is clear that $K$ may be increased so that it will also hold for the smaller positive values of $n$. Thus our theorem is proved when $l=0$.

Passing now to the case $l \neq 0$, we note first that by a theorem of STURM* between two successive roots of (24) there lies one and only one root of (1). From this, together with the fact that the first inequality (23) holds when $l=0$, we infer at once the truth of this formula when $l \neq 0$.

Reasoning now on equation (22) precisely as we reasoned above on (26), we see that however small $\delta$ may be chosen, all the large roots of (1) lie in the intervals

$$
\left|\frac{2 \nu+3}{4} \pi+\frac{\pi}{2}+k \pi-\lambda\right|<\delta \quad(k=1,2, \cdots),
$$

and since these intervals for small values of $\delta$ alternate with the intervals (27) we see from the theorem of STURM last referred to that for large values of $k$ one and only one root of (1) lies in each interval (29).

That furthermore the large roots of

$$
\cos \left(\lambda-\frac{2 \nu+3}{4} \pi\right)+\frac{8(\nu l+h)-l\left(4 \nu^{2}+8 \nu+3\right)}{8 l \lambda} \sin \left(\lambda-\frac{2 \nu+3}{4} \pi\right)=0
$$

also all lie in the intervals (29) is obvious; that one and only one of them lies in each of these intervals follows from the fact that the distance between two successive roots approaches $\pi$ as its limit, as we see from Lemma 1 .

Hence all the large roots of $(30)$ coincide very nearly with the large roots of (22).

From here on the reasoning is identical with the reasoning in the case $l=0$; and our theorem is established.

CoRollaRY. If we represent by $\lambda_{1}, \lambda_{2}, \lambda_{3}, \ldots$ the successive positive roots of equation (1), then there exists a constant $q$ and a positive constant $L$ such that

$$
\left|\lambda_{n}-n \pi-q\right|<\frac{L}{n} \quad(n=1,2,3, \cdots) .
$$

Let us consider the series

$$
\sum_{n=1}^{\infty}\left[\lambda_{n+1}-\lambda_{n}-\pi\right]
$$

which by Theorem $I$ is absolutely convergent.

If we represent the value to which it converges by $q_{1}$ and its remainder after $(n-1)$ terms by $R_{n}$, we have

\footnotetext{
${ }^{*}$ Loc. cit., \& XIX.
}

$$
\lambda_{n}-\lambda_{1}-(n-1) \pi+R_{n}=q_{1},
$$


and consequently

$$
\begin{aligned}
&\left|\lambda_{n}-n \pi+\pi-\lambda_{1}-q_{1}\right|=\left|R_{n}\right|<\sum_{n=n}^{\infty} \frac{K}{n^{2}} \\
&<\int_{n-1}^{\infty} \frac{K}{x^{2}} d x=\frac{K}{n} \cdot \frac{n}{n-1} \leqq \frac{2 K}{n} \quad(n \geqq 2) .
\end{aligned}
$$

Hence if we take $q=q_{1}+\lambda_{1}-\pi$, and $L \geqq 2 K$ sufficiently large to satisfy the inequality (31) for $n=1$, the truth of our corollary follows.

\section{§2. Summability and uniform summability of the development of an arbitrary function in terms of Bessel functions.}

We begin this section with a definition of summability and uniform summability and the statement of a few general facts with regard to summable and uniformly summable series.

Definition. If $u_{1}(x), u_{2}(x), \ldots$ are functions of $x$, and we let

$$
\begin{aligned}
& s_{n}(x)=u_{1}(x)+u_{2}(x)+\cdots+u_{n}(x), \\
& S_{n}(x)=\frac{s_{1}(x)+s_{2}(x)+\cdots+s_{n}(x),}{n} ;
\end{aligned}
$$

then if for a particular value of $x, S_{n}(x)$ approaches a limit $U(x)$ as $n$ becomes infinite, the series

$$
u_{1}(x)+u_{2}(x)+\cdots
$$

is said to be summable to the value $U(x)$; if $S_{n}(x)$ upproaches $U(x)$ uniformly throughout a certain interval, the series is said to be uniformly summable.*

It is a well known fact that every convergent series is summable, and every uniformly convergent series is uniformly summable, to the values to which they are convergent or uniformly convergent, respectively, but that the converse is not true. Moreover, the sum of any two summable or uniformly summable series is summable or uniformly summable, respectively, and its value is equal to the sum of the values of the original series.

We will now proceed to establish some sufficient conditions that the series

$$
\sum_{n=1}^{\infty} J_{\nu}\left(\lambda_{n} x\right) \frac{\int_{0}^{1} x f(x) J_{\nu}\left(\lambda_{n} x\right) d x}{\int_{0}^{1} x\left[J_{\nu}\left(\lambda_{n} x\right)\right]^{2} d x},
$$

where $\lambda_{1}, \lambda_{2}, \ldots$ are the successive positive roots of equation (1), should be

* The term summable is often used in other more general senses. With these we shall not be concerned in this paper. 
summable at every point of the interval $0<x<1$ at which the function $f(x)$, which appears in the formulæ for the coefficients, is continuous or has a finite jump, and should be uniformly summable throughout every closed sub-interval lying within an interval of continuity of $f(x)$. The series (35) is the ordinary formal development of an arbitrary function $f(x)$ in terms of Bessel functions.

We begin by proving two lemmas.

Lемм 4. If we represent by $\lambda_{1}, \lambda_{2}, \cdots$ the successive positive roots of equation (1), then the series

$$
\sum_{n=1}^{\infty} \frac{1}{\lambda_{n}^{\rho}} \int_{0}^{1} \phi(x) \sin \left(\lambda_{n} x-\alpha\right) d x^{*}
$$

where $\alpha$ is any constant, will be absolutely convergent, provided $\phi(x)$ is finite and integrable.

Using the Law of the Mean and the corollary of Theorem I we obtain the formula

$$
\begin{aligned}
\left|\int_{0}^{1} \phi(x) \sin \left(\lambda_{n} x-\alpha\right) d x-\int_{0}^{1} \phi(x) \sin \{(n \pi+q) x-\alpha\} d x\right| \\
=\left|\int_{0}^{1} \phi(x)(\cos \xi)\left(\lambda_{n}-n \pi-q\right) x d x\right|<\frac{L}{n} \int_{0}^{1}|\phi(x)| d x=\frac{L_{1}}{n},
\end{aligned}
$$

where $L_{1}$ is a positive constant. We may therefore write

$$
\begin{aligned}
& \left|\frac{1}{\lambda_{n}^{\rho}} \int_{0}^{1} \phi(x) \sin \left(\lambda_{n} x-\alpha\right) d x\right| \\
& \leqq \frac{1}{\lambda_{n}^{\rho}}\left\{\left|\int_{0}^{1} \phi(x)\left[\sin \left(\lambda_{n} x-\alpha\right)-\sin \{(n \pi+q) x-\alpha\}\right] d x\right|\right. \\
& \left.+\left|\int_{0}^{1} \phi(x) \sin \{(n \pi+q) x-\alpha\} d x\right|\right\} \\
& <\frac{L_{1}}{n \lambda_{n}^{\rho}}+\frac{1}{\lambda_{n}^{\rho}}\left|\int_{0}^{1} \phi(x) \sin \{(n \pi+q) x-\alpha\} d x\right| .
\end{aligned}
$$

But by Theorem I

$$
\frac{L_{1}}{n \lambda_{n}^{\rho}}<\frac{L_{1}}{C^{\rho} n^{1+\rho}},
$$

and hence the series whose general term is the first term on the right hand side of (37) is convergent. If now we can show that the series which has for its general term the second term on the right hand side of (37) is convergent, it will follow that the series (36) is absolutely convergent.

* We have taken the sine as the trigonometric function appearing in the integrand. That the lemma holds equally well for the cosine is seen by making the change of variable

$$
\alpha=\alpha^{\prime}-\frac{\pi}{2} \text {. }
$$


We have

$$
\int_{0}^{1} \phi(x) \sin \{(n \pi+q) x-\alpha\} d x=u_{n}+v_{n}
$$

where

$u_{n}=\int_{0}^{1} \phi(x) \cos (q x-\alpha) \sin n \pi x d x, \quad v_{n}=\int_{0}^{1} \phi(x) \sin (q x-\alpha) \cos n \pi x d x$.

These quantities $u_{n}$ and $v_{n}$ are constant multiples of Fourier's constants belonging to the functions $\phi(x) \cos (q x-\alpha)$ and $\phi(x) \sin (q x-\alpha)$, respectively. Therefore, since these functions are finite and integrable, it follows from a theorem due to de LA Vallé Poussin that

$$
\sum_{n=1}^{\infty} u_{n}^{2}, \quad \sum_{n=1}^{\infty} v_{n}^{2}
$$

are convergent.* Hence the series

$$
\sum_{n=1}^{\infty} \frac{\left|u_{n}\right|}{n^{\rho}}, \quad \sum_{n=1}^{\infty} \frac{\left|v_{n}\right|}{n^{\rho}}
$$

are convergent. $\dagger$

But we have from (38) and Theorem I

(40) $\frac{1}{\lambda_{n}^{\rho}}\left|\int_{0}^{1} \phi(x) \sin \{(n \pi+q) x-\alpha\} d x\right| \leqq \frac{\left|u_{n}\right|}{\lambda_{n}^{\rho}}+\frac{\left|v_{n}\right|}{\lambda_{n}^{\rho}} \leqq \frac{\left|u_{n}\right|}{C^{\rho} n^{\rho}}+\frac{\left|v_{n}\right|}{C^{\rho} n^{\rho}}$.

Since the two series in (39) are convergent, it follows from (40) that the series whose general term is the left hand side of $(40)$ is convergent, and our lemma is proved.

Lемма 5. If the general term of the series (35) is written in the form

$$
\begin{aligned}
& \frac{2}{\sqrt{x}} \cos (q x-\alpha) \cos n \pi x \int_{0}^{1} v^{\prime} x f(x) \cos (q x-\alpha) \cos n \pi x d x \\
+ & \frac{2}{\sqrt{x}} \sin (q x-\alpha) \sin n \pi x \int_{0}^{1} \sqrt{x} f(x) \sin (q x-\alpha) \sin n \pi x d x+v_{n}(x),
\end{aligned}
$$

then, provided the function $f(x)$ is finite and integrable, the series

$$
\sum_{n=1}^{\infty} v_{n}(x)
$$

will be uniformly summable in every interval

$$
c \leqq x \leqq 1
$$

*Annales de la Société Scientifique de Bruxelles, vol. 17 (1892-3); also. Bôcher, Annals of Mathematics, ser. 2, vol. 7 (1906), p. 107.

† Bôcher, loc. cit., p. 108, lemma. This lemma is there proved only for the case $\rho=1 \mathrm{but}$, the proof applies equally well to the general case $\rho>\frac{1}{2}$. 
for which

and if we write

$$
0<c<1 \text {, }
$$

the series

$$
\begin{aligned}
& \bar{v}_{n}(x)=\dot{x}^{3} v_{n}(x) \\
& \bar{v}_{n}(0)=\lim _{x=+0} \bar{v}_{n}(x)
\end{aligned}
$$

$$
\sum_{n=1}^{\infty} \bar{v}_{n}(x)
$$

will be uniformly summable and its terms will be continuous in the intervai

$$
0 \leqq x \leqq 1 .
$$

It is obvious that it will be sufficient to prove only the second part of the above lemma, since the first part is an immediate consequence of the second. We will show then that the facts stated hold with regard to the series whose general term is $\bar{v}_{n}(x)$, and it will follow at once that the facts stated hold also with regard to the series whose general term is $v_{n}(x)$.

In view of the asymptotic expansion of $J_{\nu}(\lambda)$ [see equation (20)] we may write

$$
J_{\nu}(\lambda)=\frac{A}{\sqrt{\lambda}} \cos (\lambda-\alpha)+\frac{\psi(\lambda)}{\lambda^{3}}, \quad J_{\nu+1}(\lambda)=\frac{A}{\sqrt{\bar{\lambda}}} \sin (\lambda-\alpha)+\frac{\psi(\lambda)}{\lambda^{\frac{3}{3}}},
$$

where $A$ and $\alpha$ are constants and we use $\psi(\lambda)$ to represent any function of $\lambda$ that is continuous for all positive values of $\lambda$, remains finite for all such values of $\lambda$, and approaches a finite limit when $\lambda$ approaches zero. It is obvious from this definition that in different formulas, and even in different parts of the same formula, $\psi(\lambda)$ may have a different meaning.

If we set $\lambda x=y$ we obtain

$$
\int_{0}^{1} x\left[J_{\nu}(\lambda x)\right]^{2} d x=\frac{1}{\lambda^{2}} \int_{0}^{\lambda} y\left[J_{\nu}(y)\right]^{2} d y .
$$

But we have*

$$
\int_{0}^{\lambda} y\left[J_{\nu}(y)\right]^{2} d y=\frac{\lambda^{2}}{2}\left\{\left[J_{\nu}(\lambda)\right]^{2}+\left[J_{\nu+1}(\lambda)\right]^{2}\right\}-\nu \lambda J_{\nu}(\lambda) J_{\nu+1}(\lambda),
$$

and hence we obtain from (44) and (45) as a value for the denominator of the fraction in (35) $\dagger$

$$
\int_{0}^{1} x\left[J_{\nu}(\lambda x)\right]^{2} d x=\frac{A^{2}}{2 \lambda}+\frac{\psi_{1}(\lambda)}{\lambda^{2}},
$$

where $\psi_{1}(\lambda)$ is used to represent any function of $\lambda$ that remains finite when

* Cf. BykRLy, An Elementary Treatise on Fourier's Series, etc., p. 224.

$\dagger$ For the sake of simplicity we use $\lambda$ instead of $\lambda_{n}$. 
$\lambda$ increases indefinitely. It is apparent from this definition that a $\psi(\lambda)$ is always a $\psi_{1}(\lambda)$, but that a $\psi_{1}(\lambda)$ may not be a $\psi(\lambda)$.

Turn now to the numerator of the fraction in (35). If we use $c$ to denote any constant lying in the interval $0<x<1$, and set $\lambda x=y$, we obtain for $\lambda>c$

$$
\int_{0}^{\frac{c}{\lambda}} x f(x) J_{\nu}(\lambda x) d x=\frac{1}{\lambda^{2}} \int_{0}^{c} y f\left(\frac{y}{\lambda}\right) J_{\nu}(y) d y=\frac{\psi_{1}(\lambda)}{\lambda^{2}} .
$$

Moreover, we have from the asymptotic expansion [see equation (20)]

$$
J_{\nu}(\lambda x)=\frac{A}{\sqrt{\lambda x}}\left[\cos (\lambda x-\alpha)+\frac{1-4 \nu^{2}}{8 \lambda x} \sin (\lambda x-\alpha)+\frac{\psi(\lambda x)}{\lambda^{2} x^{2}}\right]
$$

From (48) we obtain

$$
\begin{aligned}
& \int_{\frac{c}{\lambda}}^{1} x f(x) J_{\nu}(\lambda x) d x=\frac{A}{\sqrt{\lambda}} \int_{\frac{c}{\lambda}}^{1} \sqrt{x} f(x) \cos (\lambda x-\alpha) d x \\
& +\frac{A^{\prime}}{\lambda^{3}} \int_{\frac{c}{\lambda}}^{1} \frac{f(x)}{\sqrt{x}} \sin (\lambda x-\alpha) d x+\frac{A}{\lambda^{\frac{3}{3}}} \int_{\frac{c}{\lambda}}^{1} \frac{\psi(\lambda x) f(x)}{x^{\frac{z}{4}}(\lambda x)^{\frac{z}{2}}} d x \\
& =\frac{A}{\sqrt{\bar{\lambda}}} \int_{0}^{1} \sqrt{x} f(x) \cos (\lambda x-\alpha) d x+\frac{A^{\prime}}{\lambda^{a}} \int_{0}^{1} \frac{f(x)}{\sqrt{x}} \sin (\lambda x-\alpha) d x \\
& -\frac{A}{\sqrt{\lambda}} \int_{0}^{\frac{c}{\lambda}} \sqrt{x} f(x) \cos (\lambda x-\alpha) d x-\frac{A^{\prime}}{\lambda^{\frac{3}{2}}} \int_{0}^{\frac{c}{\lambda}} \frac{f(x)}{\sqrt{x}} \sin (\lambda x-\alpha) d x \\
& +\frac{A}{\lambda^{z}} \int_{\frac{c}{\lambda}}^{1} \frac{\psi(\lambda x) f(x)}{x^{z}(\lambda x)^{z}} d x \\
& =\frac{A}{\sqrt{\bar{\lambda}}} \int_{0}^{1} \sqrt{x} f(x) \cos (\lambda x-\alpha) d x+\frac{A^{\prime}}{\lambda^{\frac{3}{2}}} \int_{0}^{1} \frac{f(x)}{\sqrt{x}} \sin (\lambda x-\alpha) d x+\frac{\psi_{1}(\lambda)}{\lambda^{\frac{1}{3}}} .
\end{aligned}
$$

Combining (47) and (49) we get

$$
\begin{aligned}
\int_{0}^{1} x f(x) J_{\nu}(\lambda x) d x=\frac{A}{\sqrt{\lambda}} \int_{0}^{1} \sqrt{x} f(x) \cos (\lambda x-\alpha) d x \\
\quad+\frac{A^{\prime}}{\lambda^{z}} \int_{0}^{1} \frac{f(x)}{v^{\prime} \bar{x}} \sin (\lambda x-\alpha) d x+\frac{\psi_{1}(\lambda)}{\lambda^{z}} .
\end{aligned}
$$

Hence, for the general term of (35) we obtain by combining (44), (46), and (50)

$$
\frac{J_{\nu}(\lambda x) \int_{0}^{1} x f(x) J_{\nu}(\lambda x) d x}{\int_{0}^{1} x\left[J_{\nu}(\lambda x)\right]^{2} d x}=\frac{P}{\frac{A^{2}}{2 \lambda}+\frac{\psi_{1}(\lambda)}{\lambda^{2}}}
$$


where

$$
\begin{aligned}
& P=\left[\frac{A}{\sqrt{\lambda x}} \cos (\lambda x-\alpha)+\frac{\psi(\lambda x)}{(\lambda x)^{\frac{3}{2}}}\right] {\left[\frac{A}{\sqrt{\lambda}} \int_{0}^{1} \sqrt{x} f(x) \cos (\lambda x-\alpha) d x\right.} \\
&\left.+\frac{A^{\prime}}{\lambda^{\frac{3}{2}}} \int_{0}^{1} \frac{f(x)}{\sqrt{x}} \sin (\lambda x-\alpha) d x+\frac{\psi_{1}(\lambda)}{\lambda^{\frac{7}{4}}}\right] .
\end{aligned}
$$

If we subtract from the right hand side of $(51)$ the two terms

$$
\begin{aligned}
& \frac{2}{\sqrt{x}} \cos (\lambda x-\alpha) \int_{0}^{1} \sqrt{x} f(x) \cos (\lambda x-\alpha) d x, \\
& \frac{2 A^{\prime}}{A \lambda \sqrt{x}} \cos (\lambda x-\alpha) \int_{0}^{1} \frac{f(x)}{\sqrt{x}} \sin (\lambda x-\alpha) d x,
\end{aligned}
$$

the quantity we have left may be written in the form

$$
\frac{\left[\frac{A \psi(\lambda x)}{\lambda^{2} x^{\frac{3}{2}}}+\frac{\psi(x, \lambda)}{\lambda^{2} \sqrt{x}}\right] \int_{0}^{1} \sqrt{x} f(x) \cos (\lambda x-\alpha) d x+\frac{\psi(\lambda x) \psi_{1}(\lambda)}{\lambda^{3} x^{\frac{3}{2}}}+\frac{\psi(x, \lambda)}{\lambda^{\frac{a}{4}} \sqrt{x}}}{\frac{A^{2}}{2 \lambda}+\frac{\psi_{1}(\lambda)}{\lambda^{2}}}
$$

where $\psi(x, \lambda)$ is used to represent any function of $x$ and $\lambda$ which for all fixed values of $\lambda$ is continuous in the interval (43) and which remains finite when $\lambda$ increases indefinitely and $x$ varies in the interval (43).

We have now broken up the general term of the series (35) into the sum of the three expressions (52), (53), and (54). It is obvious that (53) and (54), when each is multiplied by $x^{\frac{3}{2}}$ and properly defined ${ }^{*}$ for $x=0$, are continuous in the interval (43); we shall show further that each of these expressions when multiplied by $x^{\frac{3}{2}}$ forms the general term of a series which is uniformly summable in that interval. Then we shall prove that if we subtract from (52) a number of expressions which when multiplied by $x^{\frac{\pi}{3}}$ and properly defined for $x=0$ are continuous in the interval (43) and form the general terms of series. that are uniformly summable in that interval, the expression we have left will be equal to the sum of the first and second terms of (41). Our lemma will thus be established.

We will begin with the proof that (54) when multiplied by $x^{3}$ forms the general term of a series that is uniformly summable in the interval (43). For values of $\lambda$ so great that

$$
\left|\frac{\psi_{1}(\lambda)}{\lambda^{2}}\right|<\frac{A^{2}}{4 \lambda}
$$

where the $\psi_{1}(\lambda)$ occurring in this inequality is the same as the $\psi_{1}(\lambda)$ in the denominator of (54), the expression (54) when multiplied by $x^{\frac{1}{2}}$ is less in absolute value than

\footnotetext{
* I. e., defined to have the value it approaches when $x$ approaches zero.
} 


$$
\begin{aligned}
\frac{4 \lambda}{A^{2}}\left[\mid\left(\frac{A \psi(\lambda x)}{\lambda^{2}}+\frac{x \psi(x, \lambda)}{\lambda^{2}}\right) \int_{0}^{1}\right. & \sqrt{x} f(x) \cos (\lambda x-\alpha) d x \mid \\
& \left.+\left|\frac{\psi(\lambda x) \psi_{1}(\lambda)}{\lambda^{3}}\right|+\left|\frac{x \psi(x, \lambda)}{\lambda^{z}}\right|\right] .
\end{aligned}
$$

Since $\sqrt{x} f(x)$ is finite and integrable in the interval $0 \leqq x \leqq 1$ it follows from Lemma 4 and the first inequality in (23) that the series whose general term is (55) is absolutely and uniformly convergent and therefore uniformly summable in the interval (43).

We will now show that (53) when multiplied by $x^{3}$ is the general term of a series that is uniformly summable in the interval (43). If we set $M=2 A^{\prime} / A$, (53) when multiplied by $x^{\frac{3}{2}}$ becomes

$$
\begin{aligned}
& \frac{M x \cos (\lambda x-\alpha)}{\lambda} \int_{0}^{c} \frac{f(x)}{\sqrt{x}} \sin (\lambda x-\alpha) d x \\
& \quad+\frac{M x \cos (\lambda x-\alpha)}{\lambda} \int_{c}^{1} \frac{f(x)}{\sqrt{x}} \sin (\lambda x-\alpha) d x .
\end{aligned}
$$

The second term in (56) is equal to

where we define

$$
\frac{M x \cos (\lambda x-\alpha)}{\lambda} \int_{0}^{1} F(x) \sin (\lambda x-\alpha) d x
$$

$$
F(x)=\frac{f(x)}{\sqrt{x}} \quad(c \leqq x \leqq 1), \quad F(x)=0 \quad(0 \leqq x<c),
$$

and hence it follows from Lemma 4 that it is the general term of a series that is absolutely and uniformly convergent and therefore uniformly summable in the interval (43).

We have next to examine the series whose general term is the first term in (56). It follows from the corollary of Theorem I that

$$
\lambda_{n}=n \pi+q+\frac{\psi_{1}\left(\lambda_{n}\right)}{n}
$$

and consequently

$$
\begin{aligned}
\cos \left(\lambda_{n} x-\alpha\right) & =\cos [(n \pi+q) x-\alpha] \cos \frac{x \psi_{1}\left(\lambda_{n}\right)}{n} \\
& -\sin [(n \pi+q) x-\alpha] \sin \frac{x \psi_{1}\left(\lambda_{n}\right)}{n} \\
& =\cos [(n \pi+q) x-\alpha]+\frac{\psi\left(x, \lambda_{n}\right)}{n} .
\end{aligned}
$$

In a similar manner we may also obtain from (57) 


$$
\sin \left(\lambda_{n} x-\alpha\right)=\sin [(n \pi+q) x-\alpha]+\frac{\psi\left(x, \lambda_{n}\right)}{n} .
$$

By the use of (58) and (59) and the first inequality in (23), the first term in (56) reduces to

$$
\frac{M x \cos [(n \pi+q) x-\alpha]}{\lambda_{n}} \int_{0}^{c} \frac{f(x)}{\sqrt{x}} \sin [(n \pi+q) x-\alpha] d x+\frac{\psi\left(x, \lambda_{n}\right)}{n^{2}}
$$

and since the second term in (60) is the general term of a series that is absolutely and uniformly convergent and therefore uniformly summable in the interval (43), we have only to examine the series whose general term is the first term in $(60)$.

Using the value of $\lambda_{n}$ given by (57), we see that if we add to the first term in (60) the term

$$
\frac{\left(q+\frac{\psi_{1}\left(\lambda_{n}\right)}{n}\right) M x \cos [(n \pi+q) x-\alpha]}{n \pi\left(n \pi+q+\frac{\psi_{1}\left(\lambda_{n}\right)}{n}\right)} \int_{0}^{c} \frac{f(x)}{\sqrt{x}} \sin [(n \pi+q) x-\alpha] d x,
$$

which is easily seen to be the general term of a series that is absolutely and uniformly convergent, and therefore uniformly summable in the interval (43), we obtain the expression

$$
\frac{M x \cos [(n \pi+q) x-\alpha]}{n \pi} \int_{0}^{c} \frac{f(x)}{\sqrt{x}} \sin [(n \pi+q) x-\alpha] d x .
$$

If now we can show that the series which has (61) as its general term is uniformly summable in the interval (43), we shall have proved that the series which has (53) multiplied by $x^{\frac{3}{3}}$ as its general term is uniformly summable in that same interval.

By a familiar trigonometric formula, (61) reduces to the form

$\frac{M x}{2 n \pi} \int_{0}^{c} \frac{f\left(x^{\prime}\right)}{\sqrt{x^{\prime}}}\left\{\sin \left[(n \pi+q)\left(x+x^{\prime}\right)-2 \alpha\right]-\sin \left[(n \pi+q)\left(x-x^{\prime}\right)\right]\right\} d x^{\prime} ;$ which, if we set $K=M / 2 \pi$, may be written in the form

$$
\begin{aligned}
K x & \int_{0}^{c} \frac{f\left(x^{\prime}\right)}{\sqrt{x^{\prime}}} \cos \left[q\left(x+x^{\prime}\right)-2 \alpha\right] \frac{\sin n \pi\left(x+x^{\prime}\right)}{n} d x^{\prime} \\
& +K x \int_{0}^{c} \frac{f\left(x^{\prime}\right)}{\sqrt{x^{\prime}}} \sin \left[q\left(x+x^{\prime}\right)-2 \alpha\right] \frac{\cos n \pi\left(x+x^{\prime}\right)}{n} d x^{\prime} \\
& -K x \int_{0}^{c} \frac{f\left(x^{\prime}\right)}{\sqrt{x^{\prime}}} \cos q\left(x-x^{\prime}\right) \frac{\sin n \pi\left(x-x^{\prime}\right)}{n} d x^{\prime} \\
& -K x \int_{0}^{c} \frac{f\left(x^{\prime}\right)}{\sqrt{x^{\prime}}} \sin q\left(x-x^{\prime}\right) \frac{\cos n \pi\left(x-x^{\prime}\right)}{n} d x^{\prime} .
\end{aligned}
$$

Trans. Am. Math. Soc. 27 
We are going to show that each term in (62) is the general term of a series that is uniformly summable in the interval (43).

If we represent by $s_{n}(z)$ the sum of the first $n$ terms of the series

$$
\sum_{n=1}^{\infty} \frac{\sin n z}{n}
$$

we have for values of $z$ such that $\sin (z / 2) \neq 0$ *

$$
\begin{aligned}
& s_{p}(z)-s_{n}(z) \\
& =\frac{\frac{1}{n+1} \cos \frac{(2 n+1) z}{2}-\sum_{\nu=n+1}^{\nu=p-1}\left(\frac{1}{\nu}-\frac{1}{\nu+1}\right) \cos \frac{(2 \nu+1) z}{2}-\frac{1}{p} \cos \frac{(2 p+1) z}{2}}{2 \sin \frac{z}{2}}
\end{aligned}
$$

In an analogous manner we obtain for the series

the relation

$$
\sum_{n=1}^{\infty} \frac{\cos n z}{n}
$$

$$
\begin{aligned}
& s_{p}(z)-s_{n}(z) \\
& =\frac{-\frac{1}{n+1} \sin \frac{(2 n+1) z}{2}+\sum_{\nu=n+1}^{\nu=p-1}\left(\frac{1}{\nu}-\frac{1}{\nu+1}\right) \sin \frac{(2 \nu+1) z}{2}+\frac{1}{p} \sin \frac{(2 p+1) z}{2}}{2 \sin \frac{z}{2}}
\end{aligned}
$$

In view of (64) we have for the series (63)

$$
\begin{aligned}
& \left|s_{p}(z)-s_{n}(z)\right| \\
& <\frac{\frac{1}{n+1}+\sum_{\nu=n+1}^{\nu=p-1}\left(\frac{1}{\nu}-\frac{1}{\nu+1}\right)+\frac{1}{p}}{2\left|\sin \frac{z}{2}\right|}=\frac{1}{(n+1) \cdot\left|\sin \frac{z}{2}\right|} \quad(p=n+1, n+2, \cdots) .
\end{aligned}
$$

In an analogous manner we obtain for the series (65) by use of the relation (66) the same inequality (67).

If now we represent by $s_{n}(x)$ the sum of the first $n$ terms of the series whose general term is the first term in (62), we obtain by means of (67), for all values of $x$ in the interval $0<x \leqq 1$, the inequality

$$
\left|s_{p}(x)-s_{n}(x)\right| \leqq K x \int_{0}^{c}\left|\frac{f\left(x^{\prime}\right)}{\sqrt{x^{\prime}}} \cos \left[q\left(x+x^{\prime}\right)-2 \alpha\right] \sum_{\nu=n+1}^{\nu=p} \frac{\sin n \pi\left(x+x^{\prime}\right)}{n}\right| d x^{\prime}
$$

\footnotetext{
* Cf. page 110 of BûCHER's article referred to above.
} 


$$
<K \int_{0}^{c} \frac{x}{x+x^{\prime}} \cdot \frac{\left|f\left(x^{\prime}\right)\right|}{\sqrt{x^{\prime}}} \cdot \frac{x+x^{\prime}}{(n+1) \cdot\left|\sin \frac{\pi\left(x+x^{\prime}\right)}{2}\right|} d x^{\prime}<\frac{K_{1}}{n+1}
$$

where $K_{1}$ is a positive constant. For $x=0$ the last inequality obviously holds, since each term of the series we are discussing is equal to zero. Hence it follows that the series whose general term is the first term in (62) is uniformly convergent and hence uniformly summable in the interval (43). In a precisely analogous manner we may establish by use of the relation (67) the fact that the series whose general term is the second term in (62) is uniformly summable in the interval (43).

We will consider next the series whose general term is the fourth term in (62). If we represent by $s_{n}(x)$ the sum of the first $n$ terms of that series we have, in view of $(67)$,

$$
\begin{aligned}
&\left|s_{p}(x)-s_{n}(x)\right| \leqq K x \int_{0}^{c}\left|\frac{f\left(x^{\prime}\right)}{\sqrt{x^{\prime}}} \sin q\left(x-x^{\prime}\right) \sum_{\nu=n+1}^{\nu=p} \frac{\cos n \pi\left(x-x^{\prime}\right)}{n}\right| d x^{\prime} \\
& \leqq K x \int^{c} \frac{\left|f\left(x^{\prime}\right)\right|}{\imath \cdot \overline{x^{\prime}}} \cdot \frac{\left|\sin q\left(x-x^{\prime}\right)\right|}{(n+1) \cdot\left|\sin \frac{\pi\left(x-x^{\prime}\right)}{2}\right|} d x^{\prime}<\frac{K_{2}}{n+1} \\
&(p=n+1, n+2, \cdots)
\end{aligned}
$$

for all values of $x$ in the interval (43), $K_{2}$ being a positive constant. Hence the series whose general term is the fourth term of (62) is uniformly convergent and therefore uniformly summable in the interval (43).

We have finally to consider the series whose general term is the third term in (62). This term may be written in the form

$$
\begin{aligned}
K x \cos q x \int_{c}^{0} \frac{f\left(x^{\prime}\right)}{\sqrt{x^{\prime}}} \cos q x^{\prime} & \frac{\sin n \pi\left(x-x^{\prime}\right)}{n} d x^{\prime} \\
& +K x \sin q x \int_{c}^{0} \frac{f\left(x^{\prime}\right)}{\sqrt{x^{\prime}}} \sin q x^{\prime} \frac{\sin n \pi\left(x-x^{\prime}\right)}{n} d x^{\prime} .
\end{aligned}
$$

By means of an integration by parts we may reduce the first term of (68) to the form

$$
K x \cos q x \frac{\sin n \pi x}{n} \int_{c}^{0} \frac{f\left(x^{\prime}\right)}{\sqrt{x^{\prime}}} \cos q x^{\prime} d x^{\prime}
$$

where we have set

$$
+\pi K x \cos q x \int_{0}^{0} F\left(x^{\prime}\right) \cos n \pi\left(x-x^{\prime}\right) d x^{\prime},
$$

$$
F\left(x^{\prime}\right)=\int_{c}^{x^{\prime}} \frac{f\left(x^{\prime}\right)}{\sqrt{x^{\prime}}} \cos q x^{\prime} d x^{\prime} \quad\left(c \geqq x^{\prime} \geqq 0\right) .
$$


If we represent by $s_{n}(x)$ the sum of the first $n$ terms of the series whose general term is the first term in (69), we have, by means of (67), for all values of $x$ in the interval $0<x \leqq 1$,

$$
\begin{array}{r}
\left|s_{p}(x)-s_{n}(x)\right| \leqq K\left|\cos q x \int_{c}^{0} \frac{f\left(x^{\prime}\right)}{\sqrt{x^{\prime}}} \cos q x^{\prime} d x^{\prime}\right| \frac{x}{(n+1) \cdot \sin \frac{\pi x}{2}}<\frac{K_{3}}{n+1} \\
(p=n+1, n+2, \cdots),
\end{array}
$$

where $K_{3}$ is a positive constant. For $x=0$ the last inequality obviously holds, since each term of the series that we are discussing is equal to zero. Hence it follows that the series whose general term is the first term in (69) is uniformly convergent and therefore uniformly summable in the interval (43).

Let us now consider the series whose general term is the second term in (69). If we set

where

$$
u_{n}(x)=\int_{0}^{2} \chi\left(x^{\prime}\right) \cos n \pi\left(x-x^{\prime}\right) d x^{\prime}
$$

$$
\begin{array}{ll}
\chi\left(x^{\prime}\right)=F\left(x^{\prime}\right) & \left(0 \leqq x^{\prime} \leqq c\right), \\
\chi\left(x^{\prime}\right)=0 & \left(c<x^{\prime} \leqq 2\right),
\end{array}
$$

the second term of (69) may be written in the form

$$
-\pi K x \cos q x u_{n}(x) \text {. }
$$

However, it follows from (71) that $u_{n}(x)$ is the general term of the Fourier development corresponding to the function $\chi(x)$, and since we know from (72) and (70) that $\chi\left(x^{\prime}\right)$ is finite and integrable in the interval $0 \leqq x \leqq 2$, it follows from FEJER's work * that for the series whose general term is $u_{n}(x)$,

$$
\left|S_{n}(x)\right|<N \quad(n=1,2,3, \cdots ; 0 \leqq x \leqq 2),
$$

where $S_{n}(x)$ is defined as in formula $(34)$ and $N$ is a positive constant. If now $\epsilon$ is given, positive and arbitrarily small, we have from (74) for the series whose general term is $(73)$ or the second term in $(69)$,

$$
\begin{array}{r}
\left|S_{p}(x)-S_{n}(x)\right| \leqq\left|S_{p}(x)\right|+\left|S_{n}(x)\right|<2 \pi K N x|\cos q x| \leqq \epsilon \\
\left(n=1,2,3, \cdots ; p=n+1, n+2, \cdots ; 0 \leqq x \leqq \frac{\varepsilon}{B}\right),
\end{array}
$$

where we have set $B=2 \pi K N$. Moreover it follows from another theorem of FEJER's $\dagger$ that the series whose general term is $u_{n}(x)$, and hence the series whose general term is (73) or the second term in (69), is uniformly summable in the interval

$$
\frac{\epsilon}{B} \leqq x \leqq 1,
$$

* Loc. cit., p. 60.

† Loc. cit., p. 60. 
since $\chi(x)$ is continuous in an interval including this interval. Hence we may ehoose $m$ so large that we have for the latter series,

$$
\left|S_{p}(x)-S_{n}(x)\right|<\epsilon \quad\left(p>n \geqq m ; \frac{\varepsilon}{B} \leqq x \leqq 1\right) .
$$

Combining (75) and (76), we see that the series whose general term is the second term in (69) is uniformly summable in the interval (43).

Since both the terms in (69) are the general terms of series that are uniformly summable in the interval (43), it follows that the series whose general term is the first term of $(68)$ is uniformly summable in that interval. In an entirely analogous manner it may be shown that the series whose general term is the second term in (68) is uniformly summable in the interval (43), and hence it follows that the series whose general term is $(68)$, or the third term in (62), is uniformly summable in that interval.

We have now shown that each term in (62) is the general term of a series that is uniformly summable in the interval (43). Hence (62), or (61), is the general term of a series that is uniformly summable in that interval and consequently, as we have pointed out before, (53) when multiplied by $x^{\frac{3}{2}}$ is the general term of a uniformly summable series in the same interval.

It remains now to consider the expression (52). It follows from (58) that if we subtract from (52) a term of the form

$$
\frac{2 \psi\left(x, \lambda_{n}\right)}{n \sqrt{x}} \int_{0}^{1} \sqrt{x} f(x) \cos \left(\lambda_{n} x-x\right) d x
$$

which when multiplied by $x^{\frac{3}{2}}$ and properly defined for $x=0$ is obviously continuous in the interval (43), and by Lemma 4 is the general term of an absolutely and uniformly convergent and therefore uniformly summable series in that same interval, we have left the expression

$$
\frac{2}{\sqrt{x}} \cos [(n \pi+q) x-\alpha] \int_{0}^{1} \sqrt{x} f(x) \cos \left(\lambda_{n} x-\alpha\right) d x .
$$

Moreover we obtain from (58)

$\cos \left(\lambda_{n} x-\alpha\right)=\cos [(n \pi+q) x-\alpha]-\frac{x \psi_{1}\left(\lambda_{n}\right)}{n} \sin [(n \pi+q) x-\alpha]+\frac{\psi\left(x, \lambda_{n}\right)}{n^{2}}$ and hence (78) reduces to

$$
\begin{aligned}
& \frac{2}{\sqrt{x}} \cos [(n \pi+q) x-\alpha] \int_{0}^{1} \sqrt{x} f(x) \cos [(n \pi+q) x-\alpha] d x \\
& -\frac{2 \psi_{1}\left(\lambda_{n}\right)}{n \sqrt{x}} \cos [(n \pi+q) x-\alpha] \int_{0}^{1} x^{\frac{3}{3}} f(x) \sin [(n \pi+q) x-\alpha] d x+\frac{\psi\left(x, \lambda_{n}\right)}{n^{2} \sqrt{x}} .
\end{aligned}
$$

The last term in (79) when multiplied by $x^{\frac{3}{2}}$ and properly defined for $x=0$ 
is obviously continuous in the interval (43) and is the general term of a series that is uniformly convergent and therefore uniformly summable in that interval. The second term in (79) when multiplied by $x^{\frac{3}{2}}$ and properly defined for $x=0$ is obviously continuous in the interval (43); that it is also the general term of a uniformly convergent and therefore uniformly summable series in that interval may be seen by referring to the proof of Lemma 4 . It is apparent from the latter part of that proof that a series whose general term has the form

$$
\frac{1}{n} \int_{0}^{1} \phi(x) \sin [(n \pi+q) x-\alpha] d x,
$$

where $\phi(x)$ is finite and integrable, is absolutely convergent. The second term of (79) when multiplied by $x^{\frac{3}{2}}$ consists of a factor of the form (80) multiplied by a factor that remains finite for all values of $\lambda$, and all values of $x$ in the interval (43), and hence this term when multiplied by $x^{\frac{3}{2}}$ is the general term of a series that is absolutely and uniformly convergent and therefore uniformly summable in the interval (43).

It only remains to consider the first term in (79) which may be broken up into the following sum of four terms:

$$
\begin{aligned}
& \frac{2}{\sqrt{x}} \cos (q x-\alpha) \cos n \pi x \int_{0}^{1} \sqrt{x} f(x) \cos (q x-\alpha) \cos n \pi x d x \\
& \quad-\frac{2}{\sqrt{x}} \cos (q x-\alpha) \cos n \pi x \int_{0}^{1} \sqrt{x} f(x) \sin (q x-\alpha) \sin n \pi x d x \\
& \quad-\frac{2}{\sqrt{x}} \sin (q x-\alpha) \sin n \pi x \int_{0}^{1} \sqrt{x} f(x) \cos (q x-\alpha) \cos n \pi x d x \\
& +\frac{2}{\sqrt{x}} \sin (q x-\alpha) \sin n \pi x \int_{0}^{1} \sqrt{x} f(x) \sin (q x-\alpha) \sin n \pi x d x .
\end{aligned}
$$

The first and fourth terms of (81) are identical with the first and second terms of (41). The second and third terms when multiplied by $x^{\frac{3}{2}}$ and properly defined for $x=0$ are obviously continuous in the interval (43); if we can show further that they form the general term of a series that is uniformly summable in that interval, our lemma will be completely established.

If we use $x^{\prime}$ as the variable of integration, the second and third terms of (81) when multiplied by $x^{\frac{3}{2}}$ may be written in the form

$$
\begin{aligned}
& -x \int_{0}^{1} \sqrt{x^{\prime}} f\left(x^{\prime}\right) \sin \left[q\left(x+x^{\prime}\right)-2 \alpha\right] \sin n \pi\left(x+x^{\prime}\right) d x^{\prime} \\
& -x \int_{0}^{1} \sqrt{x^{\prime}} f\left(x^{\prime}\right) \sin q\left(x-x^{\prime}\right) \sin n \pi\left(x-x^{\prime}\right) d x^{\prime} .
\end{aligned}
$$


Since, however,

$$
\begin{array}{r}
\sum_{n=1}^{n} \sin n z=\frac{\sin z-\sin (n+1) z+\sin n z}{2-2 \cos z}, \\
\frac{\sum_{n=1}^{n}\left(\sum_{n=1}^{n} \sin n z\right)}{n}=\frac{(n+1) \sin z-\sin (n+1) z}{2 n(1-\cos z)},
\end{array}
$$

we obtain for the series whose general term is the expression (82)

$$
\begin{aligned}
& S_{n}(x) \\
& =-x \int_{0}^{1} \sqrt{x^{\prime}} f\left(x^{\prime}\right) \sin \left[q\left(x+x^{\prime}\right)-2 \alpha\right] \frac{(n+1) \sin \pi\left(x+x^{\prime}\right)-\sin (n+1) \pi\left(x+x^{\prime}\right)}{4 n \sin ^{2} \pi \frac{x+x^{\prime}}{2}} d x^{\prime} \\
& \quad-x \int_{0}^{1} \sqrt{x^{\prime} f\left(x^{\prime}\right) \sin q\left(x-x^{\prime}\right) \frac{(n+1) \sin \pi\left(x-x^{\prime}\right)-\sin (n+1) \pi\left(x-x^{\prime}\right)}{4 n \sin ^{2} \pi \frac{x-x^{\prime}}{2}} d x^{\prime},}
\end{aligned}
$$

where $S_{n}(x)$ is defined as in formula (34).

We are going to show that as $n$ becomes infinite each of the terms on the right hand side of (83) approaches a limit uniformly for all values of $x$ in the interval (43) and consequently that the series whose general term is (82), or the second and third terms in (81) multiplied by $x^{\frac{3}{2}}$, is uniformly summable in that interval.

Consider first the second term on the right hand side of (83). It may be written

$$
\begin{aligned}
& -\frac{x}{4} \cdot \frac{n+1}{n} \int_{0}^{1} \frac{\sin q\left(x-x^{\prime}\right)}{\sin \pi \frac{x-x^{\prime}}{2}} \sqrt{x^{\prime}} f\left(x^{\prime}\right) \frac{\sin \pi\left(x-x^{\prime}\right)}{\sin \pi \frac{x-x^{\prime}}{2}} d x^{\prime} \\
& +\frac{x}{4} \cdot \frac{n+1}{n} \int_{0}^{1} \frac{\sin q\left(x-x^{\prime}\right)}{\sin \pi \frac{x-x^{\prime}}{2}} \cdot \frac{\sqrt{x^{\prime}} f\left(x^{\prime}\right)\left(x-x^{\prime}\right)}{\sin \pi \frac{x-x^{\prime}}{2}} \cdot \frac{\sin (n+1) \pi\left(x-x^{\prime}\right)}{(n+1) \sqrt{x-x^{\prime}}} \cdot \frac{d x}{V^{\prime} x-x^{\prime}}
\end{aligned}
$$

The integrand of the integral in the first term of (84) consists of a function of $x$ and $x^{\prime}$ that is continuous in both variables multiplied into a function of $x^{\prime}$ that is finite and integrable in the interval of integration. Hence that integral defines a function of $x$ that is continuous and therefore finite in the interval (43), and consequently the first term in (84) approaches a limit uniformly for all values of $x$ in the interval (43) as $n$ becomes infinite.

The factor

$$
\frac{\sin (n+1) \pi\left(x-x^{\prime}\right)}{(n+1) \sqrt{x-x^{\prime}}}
$$

which occurs in the integrand of the second term in (84) approaches zero uni- 
formly for all values of $x$ and $x^{\prime}$ as $n$ becomes infinite. For, given $\epsilon$ positive and arbitrarily small, we have for values of $x$ and $x^{\prime}$ such that

$$
\begin{gathered}
\left|x-x^{\prime}\right|<\frac{\epsilon^{2}}{\pi^{2}}, \\
\left|\frac{\sin (n+1) \pi\left(x-x^{\prime}\right)}{(n+1) \sqrt{x-x^{\prime}}}\right| \leqq \frac{(n+1) \pi\left|x-x^{\prime}\right|}{(n+1) \sqrt{\left|x-x^{\prime}\right|}}=\pi \sqrt{\left|x-x^{\prime}\right|}<\epsilon .
\end{gathered}
$$

Moreover, we can choose $m$ so large that for all values of $x$ and $x^{\prime}$ such that

$$
\left|x-x^{\prime}\right| \geqq \frac{\epsilon^{2}}{\pi^{2}}
$$

we have

$$
\left|\frac{\sin (n+1) \pi\left(x-x^{\prime}\right)}{(n+1) \sqrt{x-x^{\prime}}}\right|<\epsilon \quad(n \geqq m) .
$$

We see then that the integral in the second term in (84) is in absolute value not greater than

$$
\begin{array}{r}
\int_{0}^{1}\left|\frac{\sin q\left(x-x^{\prime}\right)}{\sin \frac{x-x^{\prime}}{2}} \cdot \frac{\sqrt{x^{\prime}} f\left(x^{\prime}\right)\left(x-x^{\prime}\right)}{\sin \pi \frac{x-x^{\prime}}{2}}\right| \cdot\left|\frac{\sin (n+1) \pi\left(x-x^{\prime}\right)}{(n+1) \sqrt{x-x^{\prime}}}\right| \cdot \frac{d x^{\prime}}{\left|\sqrt{x-x^{\prime}}\right|} \\
<K \epsilon \int_{0}^{1} \frac{d x^{\prime}}{\mid \sqrt{x-x^{\prime} \mid} \quad(n \geqq m ; 0 \leqq x \leqq 1)},
\end{array}
$$

where $K$ is a positive constant. But

$$
\int_{0}^{1} \frac{d x^{\prime}}{\left|\sqrt{x-x^{\prime}}\right|}=\int_{-x}^{1-x} \frac{d \alpha}{\sqrt{|\alpha|}}<2 \int_{0}^{1} \frac{d \alpha}{\sqrt{\alpha}}=4 .
$$

Combining (87) with (86) we see that the integral in the second term in (84) approaches zero uniformly for all values of $x$ in the interval (43) as $n$ becomes infinite, and hence the second term itself approaches zero uniformly in the same interval.

The first term on the right hand side of (83) may be broken up into the sum of the following three integrals :

$$
\begin{aligned}
& -\frac{x}{4} \int_{0}^{1} \frac{\sin \left[q\left(x+x^{\prime}\right)-2 \alpha\right]}{\sin \pi \frac{x+x^{\prime}}{2}} \sqrt{x^{\prime}} f\left(x^{\prime}\right) \frac{\sin \pi\left(x+x^{\prime}\right)}{\sin \pi \frac{x+x^{\prime}}{2}} d x^{\prime}, \\
& -\frac{x}{4 n} \int_{0}^{1} \frac{\sin \left[q\left(x+x^{\prime}\right)-2 \alpha\right]}{\sin \pi \frac{x+x^{\prime}}{2}} \sqrt{x^{\prime}} f\left(x^{\prime}\right) \frac{\sin \pi\left(x+x^{\prime}\right)}{\sin \pi \frac{x+x^{\prime}}{2}} d x^{\prime}, \\
& +\frac{x}{4} \int_{0}^{1} \frac{\sin \left[q\left(x+x^{\prime}\right)-2 \alpha\right]}{\sin \pi \frac{x+x^{\prime}}{2}} \sqrt{x^{\prime}} f\left(x^{\prime}\right) \frac{\sin (n+1) \pi\left(x+x^{\prime}\right)}{n \sin \pi \frac{x+x^{\prime}}{2}} d x^{\prime},
\end{aligned}
$$


provided that we can show that each of these integrals converges for all values of $x$ in the interval (43). For this purpose we must consider the values of $q$.

By comparing equations (20), (44), and (22) we see that (1) may be written $\frac{A\left[8(\nu l+h)-l\left(4 \nu^{2}+8 \nu+3\right)\right]}{8 \lambda} \cos (\lambda-\alpha)-A l \sin (\lambda-\alpha)+\frac{\psi(\lambda)}{\lambda^{2}}=0$.

Consequently, except in the case $l=0$, the large roots of this equation coincide more and more nearly with the values

$$
\alpha+k \pi,
$$

where $k$ takes on large positive integral values. In the exceptional case $l=0$ they coincide more and more nearly with

$$
\frac{\pi}{2}+\alpha+k \pi \text {. }
$$

Since, by (31), we see that $\lambda_{n}$ coincides more and more nearly with $q+n \pi$, it follows that according as the $l$ of equation (1) is or is not zero,

$$
q=\frac{\pi}{2}+\alpha+k_{1} \pi \quad \text { or } \quad q=\alpha+k_{2} \pi,
$$

where $k_{1}$ and $k_{2}$ are positive or negative integers or zero. Consequently the expression

$$
\frac{\sin \left[q\left(x+x^{\prime}\right)-2 \alpha\right]}{\sin \pi \frac{x+x^{\prime}}{2}}
$$

approaches a finite limit as $x+x^{\prime}$ approaches the limit 2, and hence the integrals in (88) converge for all values of $n$ and all values of $x$ in the interval (43).

It now remains to be shown that the expression (88) approaches a limit uniformly for all values of $x$ in the interval (43), as $n$ becomes infinite. The first term of that expression is independent of $n$ and hence need not be considered further. The integral in the second term has for its integrand the product of a function of $x$ and $x^{\prime}$ that is continuous in both variables and a function of $x^{\prime}$ that is finite and integrable. It therefore defines a continuous function of $x$, and consequently the second term approaches zero uniformly for all values of $x$ in the interval (43), as $n$ becomes infinite.

The same is true of the third term of (88). For, since the expression

$$
\frac{\sin (n+1) \pi\left(x+x^{\prime}\right)}{n \sin \pi\left(\frac{x+x^{\prime}}{2}\right)}
$$

remains finite for all positive integral values of $n$ and all values of $x$ and $x^{\prime}$, it 
follows that if $\epsilon$ is given positive and arbitrarily small, we can choose $\eta$ so small that

$$
\begin{array}{r}
\left|\frac{x}{4} \int_{1-\eta}^{1} \frac{\sin \left[q\left(x+x^{\prime}\right)-2 \alpha\right]}{\sin \pi \frac{x+x^{\prime}}{2}} \sqrt{x^{\prime}} f\left(x^{\prime}\right) \frac{\sin (n+1) \pi\left(x+x^{\prime}\right)}{n \sin \pi \frac{x+x^{\prime}}{2}} d x^{\prime}\right|<\frac{\epsilon}{2} . \\
(n=1,2,3, \cdots ; 0 \leqq x \leqq 1) .
\end{array}
$$

We can then choose $\delta$ so small that

$$
\left|\frac{x}{4} \int_{0}^{1-\eta} \frac{\sin \left[q\left(x+x^{\prime}\right)-2 \alpha\right]}{\sin \pi \frac{x+x^{\prime}}{2}} \sqrt{x^{\prime} f\left(x^{\prime}\right)} \frac{\sin (n+1) \pi\left(x+x^{\prime}\right)}{n \sin \pi \frac{x+x^{\prime}}{2}} d x^{\prime}\right|<\frac{\epsilon}{2}
$$$$
(n=1,2,3, \cdots ; 0 \leqq x<\delta) \text {. }
$$

Finally, $\eta$ and $\delta$ being fixed, we can choose $m$ so large that

$$
\left|\frac{x}{4 n} \int_{0}^{1-\eta} \frac{\sin \left[q\left(x+x^{\prime}\right)-2 \alpha\right]}{\sin \pi \frac{x+x^{\prime}}{2}} \sqrt{x^{\prime}} f\left(x^{\prime}\right) \frac{\sin (n+1) \pi\left(x+x^{\prime}\right)}{\sin \pi \frac{x+x^{\prime}}{2}} d x^{\prime}\right|<\frac{\epsilon}{2}
$$

Combining (92), (91), and (90) we see that the third term of (88) approaches zero uniformly for all values of $x$ in the interval (43) as $n$ becomes infinite.

We have now shown that as $n$ becomes infinite each of the terms on the right hand side of (83) approaches a limit uniformly for all values of $x$ in the interval (43) and therefore it follows that the series whose general term is (82), or the second and third terms of (81) multiplied by $x^{3}$, is uniformly summable in the interval (43). Hence, as we have pointed out above, our lemma is proved.

We are now prepared to prove the following theorem:

Theorem II. If in the interval $0 \leqq x \leqq 1$ the function $f(x)$ is finite and integrable, then the series (35) will be summable at every point of the interval $0<x<1$ at which $f(x)$ is continuous or has a finite jump; and will be uniformly summable in every closed interval lying in that interval which does not include a point of discontinuity of $f(x)$.

Since $f(x)$ is finite and integrable,

$$
\sqrt{x} f(x) \cos (q x-\alpha) \quad \text { and } \quad \sqrt{x} f(x) \sin (q x-\alpha)
$$

are finite and integrable, and hence it follows from a theorem due to FEJER* that the two series whose general terms are the first and second terms of (41), respectively, are summable at every point of the interval $0<x<1$ at which the function $f(x)$ is continuous or has a finite jump, these series being essentially equivalent to the cosine development and sine development, respectively, of $\sqrt{x} f(x) \cos (q x-\alpha)$ and $\sqrt{x} f(x) \sin (q x-\alpha)$. We know from Lemma 5

\footnotetext{
* Loo. cit., p. 53.
} 
that the series which has for its general term the third term of $(41)$ is uniformly summable throughout the interval (42), and hence, since the $c$ of that interval is arbitrary, is summable at every point of the interval $0<x<1$. Consequently the series (35), whose general term is the sum of the three terms occurring in (41), is summable at every point of the interval $0<x<1$ at which $f(x)$ is continuous or has a finite jump.

We know further from FEJER's work * that the series having for their general terms the first two terms in (41) are uniformly summable in every closed interval which lies in the interval $0<x<1$ and does not include a point of discontinuity of $f(x)$, and it follows from Lemma 5 that the series having for its general term the third term in (41) is uniformly summable in any such interval. The series (35) is therefore uniformly summable in any such interval and our theorem is completely established.

Conollary. If we define $S_{n}(x)$ as in formula (34), we have for the series (35)

$$
\left|S_{n}(x)\right|<M \quad(n=1,2,3, \cdots ; 0<c \leqq x \leqq 1)
$$

where $M$ is a positive constant.

For each of the two series whose general terms are the first and second terms of (41) we have from FEJER's work $†$

$$
\left|S_{n}(x)\right|<M_{1} \quad(n=1,2,3, \cdots ; 0<c \leqq x \leqq 1),
$$

where $M_{1}$ is a positive constant; for the series whose general term is the third term of (41) we have from Lemma 5

$$
\left|S_{n}(x)\right|<M_{2} \quad(n=1,2,3, \cdots ; 0<c \leqq x \leqq 1),
$$

where $M_{2}$ is a positive constant. If we combine (93) and (94) the proof of our corollary follows at once.

Before proceeding to the proof of the next theorem, we wish to make the following convention. Let

$$
\phi(x)=\cos ^{2}(q x-\alpha) f(x)+\sin ^{2}(q x-\alpha) f(x)+\sum_{n=1}^{\infty} v_{n}(x),
$$

where $v_{n}$ has the same meaning as in (41), and the series is to be evaluated as a summable series. The function $\phi(x)$ we will designate as the function associated with the series (35). It will be seen that $\phi(x)$ is thus defined at all points where $f(x)$ is defined, whereas the series (35) is not necessarily summable at points where $f(x)$ is discontinuous. At points where $f(x)$ is continuous, however, it is readily seen by a reference to Lemma 5 that the series (35) is summable to precisely the value $\phi(x)$.

\footnotetext{
${ }^{*}$ Loc. cit., p. 60.

† Loc. cit., p. 60.
} 
Theorem III. If we multiply the series (35) by any function $\chi(x)$ that is finite and integrable in the interval $0 \leqq x \leqq 1$, and integrate the resulting series term by term from $\delta *$ to 1 , we shall obtain a series which is summable to the value

$$
\int_{\delta}^{1} \phi(x) \chi(x) d x
$$

where $\phi(x)$ is the function associated with the series (35).

Since the series whose general terms are the first and second terms of (41) differ from Fourier developments of finite and integrable functions only by factors which are continuous functions of $x$ in the interval $\delta \leqq x \leqq 1$, we know from a theorem due to DE LA VALLEE Poussin $\dagger$ that we can multiply each of them by any finite and integrable function $\chi(x)$ and integrate the resulting series term by term from $\delta$ to 1 , and that the series thus obtained will converge to the value of the integral of the product of $\chi(x)$ and the function of which the series is a development multiplied by the function which appears as a factor of the series.

Since, from Lemma 5, the series whose general term is the third term of (41) is uniformly summable in the interval (42), we can multiply it by any finite and integrable function $\chi(x)$ and integrate it term by term from $\delta$ to 1 , and the resulting series will be summable to the value of the integral of the product of $\chi(x)$ and the function to which the series is summable. Thus our theorem is proved.

\section{§3. Value of the development.}

The series (35) is usually given as the formal development of an arbitrary function in terms of Bessel functions, but there are cases in which this series, even if it is convergent or summable, will not have the desired value, an extra term being necessary in order that the development may represent the function developed. $\ddagger$ However, in these cases the extra term appears naturally as part of the formal development if we keep in mind the method by which the formal development is commonly deduced.

In the problems of Mathematical Physics in which we wish to develop an arbitrary function in terms of certain normal functions, the latter functions appear as solutions of some ordinary differential equation. In the case in which the normal functions are Bessel functions the differential equation is usually

$$
\frac{d^{2} u}{d x^{2}}+\frac{1}{x} \frac{d u}{d x}+\left(\lambda^{2}-\frac{\nu^{2}}{x^{2}}\right) u=0
$$

* The quantity $\delta$ is any positive quantity less than 1 .

$\nmid$ Cf. Annales de la Société Scientifique de Bruxelles, vol. 17 (1892-3). Cf. also BôcHER's article referred to above, p. 118, footnote.

$\ddagger$ Cf. Dini : Serie di Fourier, and Bridgman, Philosophical Magazine, vol. 16 (1908), p. 947 . 
where $\nu$ is a constant $\geqq 0$ and $\lambda$ is a real constant (which may obviously be taken $\geqq 0$ ) to be determined in such a way that (97) has a solution, not identically zero, which remains finite when $x=0$, and at the point $x=1$ satisfies the condition

$$
\left[l u^{\prime}(x)+h u(x)\right]_{x=1}=0
$$

where $l$ and $h$ are given constants not both zero.

If in (97) $\lambda \neq 0$, the most general solution which remains finite at $x=0$ is

$$
u=C J_{\nu}(\lambda x),
$$

and in order that this should satisfy (98) it is necessary and sufficient that

$$
l \lambda J_{\nu}^{\prime}(\lambda)+h J_{\nu}(\lambda)=0 .
$$

This is the equation for determining the values of $\lambda$ greater than zero which we have to use. When, however, $\lambda=0$, formula (99) no longer gives the general solution of (97) which remains finite when $x=0$, this solution then being

$$
u=C x^{\nu} .
$$

The latter solution satisfies (98) when and only when

$$
l \nu+h=0 \text {. }
$$

It is, therefore, only in this case that the value $\lambda=0$ needs to be considered at all. In all other cases we develop our function in the form

$$
f(x)=\sum_{n=1}^{\infty} A_{n} J_{\nu}\left(\lambda_{n} x\right),
$$

where $\lambda_{1}, \lambda_{2}, \ldots$ are the successive positive roots of (100), and the coefficients $A$ are to be determined by the ordinary formulas.

In the exceptional case (102) the $\lambda$ 's greater than zero are still determined by $(100)$, which now reduces to

$$
-l J_{\nu+1}(\lambda)=0 . *
$$

But we also have to use the value $\lambda=0$, and thus we are led to the development

$$
f(x)=A_{1} x^{\nu}+\sum_{n=2}^{\infty} A_{n} J_{\nu}\left(\lambda_{n} x\right),
$$

where $\lambda_{2}, \lambda_{3}, \cdots$ are the positive roots of (103). Here not only are $\sqrt{x} J_{\nu}\left(\lambda_{i} x\right)$ and $\sqrt{x} J_{\nu}\left(\lambda_{j} x\right)$ orthogonal to each other, i. e.,

$$
\int_{0}^{1} x J_{\nu}\left(\lambda_{i} x\right) J_{\nu}\left(\lambda_{j} x\right) d x=0,
$$

* Cf. for instance BYERLY, loc. cit., p. 223, formula (6). 
but $x^{\nu+\frac{1}{2}}$ is also orthogonal to each of the functions $\sqrt{x} J_{\nu}\left(\lambda_{n} x\right)$, i. e.,

$$
\int_{0}^{1} x^{\nu+1} J_{\nu}\left(\lambda_{n} x\right) d x=0 \text {. }
$$

Accordingly here also the coefficients can be determined in the ordinary way. For instance, to get $A_{1}$ we multiply (104) through by $x^{\nu+1}$ and integrate term by term from 0 to 1 , thus obtaining *

so that

$$
\int_{0}^{1} x^{\nu+1} f(x) d x=A_{1} \int_{0}^{1} x^{2 \nu+1} d x=\frac{A_{1}}{2 \nu+2}
$$

$$
A_{1}=(2 \nu+2) \int_{0}^{1} x^{\nu+1} f(x) d x .
$$

If then, in developing our arbitrary function, we use all of the normal functions that satisfy (97) and (98) we shall naturally have an extra term in the case in which (102) is satisfied. Hence, what appears to be an exceptional case is really not exceptional at all, save in regard to the notation ordinarily used for expressing the solutions of (97) in terms of Bessel functions. Moreover, it is possible to adopt a notation which avoids the awkwardness of making a special case of the solution (101). For, if we set

so that

then

$$
F_{\nu}(\lambda, x)=\left(\frac{x}{2}\right)^{\nu} \sum_{i=0}^{\infty} \frac{(-1)^{i}(\lambda x)^{2 i}}{2^{2 i} i !} \frac{1}{\Gamma(\nu+i+1)}
$$

$$
J_{\nu}(\lambda x) \equiv \lambda^{\nu} F_{\nu}(\lambda, x),
$$

$$
u=C F_{\nu}(\lambda, x)
$$

will be the most general solution of (97) which remains finite when $x=0$, not only when $\lambda>0$ but also when $\lambda=0$, since in this last case it reduces to the form (101).

We now consider the series

$$
\sum_{n=1}^{\infty} F_{\nu}\left(\lambda_{n}, x\right) \frac{\int_{0}^{1} x f(x) F_{\nu}\left(\lambda_{n}, x\right) d x}{\int_{0}^{1} x\left[F_{\nu}\left(\lambda_{n}, x\right)\right]^{2} d x}=\sum_{n=1}^{\infty} A_{n} F_{\nu}\left(\lambda_{n}, x\right),
$$

where $\lambda_{1}, \lambda_{2}, \lambda_{3}, \cdots$ are the roots, positive or zero, of the equation

$$
\left[l \frac{\partial}{\partial x} F_{\nu}\left(\lambda_{1} x\right)+h F_{\nu}\left(\lambda_{1} x\right)\right]_{x=1}=0 \dagger
$$

* It is understood of course that we are using here merely the formal device ordinarily employed for finding the coefficients of the development without entering into the question of whether or not we have a right to integrate the series term by term.

† The positive roots of this equation are obviously identical with the positive roots of equation. (1), and hence all that we have proved for the latter roots holds for the former ones. 
arranged in increasing order of magnitude. The equation (107) will have a zero root when and only when (102) is fulfilled, and thus the series (106) will give the formal development of the arbitrary function $f(x)$ for all cases. This series will be identical with (35) except when (102) is fulfilled, and in that case will differ from it only in having an extra term. Since, however, this term will be finite and continuous it will not affect the summability or uniform summability of (35) and all that we have proved in the previous section with regard to that series will hold in all essential respects with regard to the series (106).

Thus if we write the general term of $(106)$ in the form

$$
\begin{aligned}
& \frac{2}{\sqrt{x}} \cos (q x-\alpha) \cos n \pi x \int_{0}^{1} \sqrt{x} f(x) \cos (q x-\alpha) \cos n \pi x d x \\
& \quad+\frac{2}{\sqrt{x}} \sin (q x-\alpha) \sin n \pi x \int_{0}^{1} \sqrt{x} f(x) \sin (q x-\alpha) \sin n \pi x d x+w_{n}(x),
\end{aligned}
$$

the series

$$
\sum_{n=1}^{\infty} w_{n}(x)
$$

will be uniformly summable in the interval $(42)$, and if we define the function

$$
\phi(x)=\cos ^{2}(q x-\alpha) f(x)+\sin ^{2}(q x-\alpha) f(x)+\sum_{n=1}^{\infty} w_{n}(x)
$$

to be the function associated with the series (106), Theorem III holds without further change if we replace the series (35) by the series (106) in the statement of that theorem. Moreover $\phi(x)$ will have the value to which the series (106) is summable at every point at which $f(x)$ is continuous.

We now wish to show that the series (106) is summable to the value

$$
\frac{1}{2}[f(x+0)+f(x-0)]
$$

at every point of the interval $0<x<1$ at which $f(x)$ is continuous or at which it has a finite jump, provided the latter points lie within an interval at every other point of which $f(x)$ is continuous. We will begin by proving some lemmas.

Lemma 6. If $f(x)$ is finite and integrable and $\phi(x)$ is the function associated with the series (106), we have

$$
\int_{0}^{1} x \phi(x) F_{\nu}\left(\lambda_{k}, x\right) d x=\int_{0}^{1} x f(x) F_{\nu}\left(\lambda_{k}, x\right) d x \quad(k=1,2,3, \cdots),
$$

where $\lambda_{1}, \lambda_{2}, \lambda_{3}, \ldots$ are the roots, positive or zero, of equation (107), arranged in increasing order of magnitude, and $F_{\nu}(\lambda, x)$ is defined by equation (105). 
We have, from the modification of Theorem III just referred to, the following equality*

$$
\begin{aligned}
\int_{\delta}^{1} x \phi & (x) F_{\nu}\left(\lambda_{k}, x\right) d x \\
& =A_{1} \int_{\delta}^{1} x F_{\nu}\left(\lambda_{1}, x\right) F_{\nu}\left(\lambda_{k}, x\right) d x+A_{2} \int_{\delta}^{1} x F_{\nu}\left(\lambda_{2}, x\right) F_{\nu}\left(\lambda_{k}, x\right) d x+\cdots
\end{aligned}
$$

Moreover $F_{\nu}\left(\lambda_{n}, x\right)$ and $F_{\nu}\left(\lambda_{k}, x\right)$ satisfy differential equations of the form (97). From these we readily deduce, if we use accents to denote differentiation with regard to $x$,

$$
\begin{aligned}
\left(\lambda_{n}^{2}-\lambda_{k}^{2}\right) & x F_{\nu}\left(\lambda_{n}, x\right) F_{\nu}\left(\lambda_{k}, x\right) \\
= & x\left[F_{\nu}\left(\lambda_{n}, x\right) F_{\nu}^{\prime \prime}\left(\lambda_{k}, x\right)-F_{\nu}\left(\lambda_{k}, x\right) F_{\nu}^{\prime \prime}\left(\lambda_{n}, x\right)\right] \\
\quad & \quad+\left[F_{\nu}\left(\lambda_{n}, x\right) F_{\nu}^{\prime}\left(\lambda_{k}, x\right)-F_{\nu}\left(\lambda_{k}, x\right) F_{\nu}^{\prime}\left(\lambda_{n}, x\right)\right] \\
= & \frac{d}{d x} x\left[F_{\nu}\left(\lambda_{n}, x\right) F_{\nu}^{\prime}\left(\lambda_{k}, x\right)-F_{\nu}\left(\lambda_{k}, x\right) F_{\nu}^{\prime \prime}\left(\lambda_{n}, x\right)\right] \\
= & \frac{d}{d x} x\left[\lambda_{n}^{2} F_{\nu}\left(\lambda_{k}, x\right) F_{\nu+1}\left(\lambda_{n}, x\right)-\lambda_{k}^{2} F_{\nu}\left(\lambda_{n}, x\right) F_{\nu+1}\left(\lambda_{k}, x\right)\right], \dagger
\end{aligned}
$$

and consequently when $\lambda_{n} \neq \lambda_{k}$

$$
\begin{aligned}
& \int_{\delta}^{l} x F_{\nu}\left(\lambda_{n}, x\right) F_{\nu}\left(\lambda_{k}, x\right) d x \\
& \quad=\frac{1}{\lambda_{n}^{2}-\lambda_{k}^{2}}\left[x\left\{\lambda_{n}^{2} F_{\nu}\left(\lambda_{k}, x\right) F_{\nu+1}\left(\lambda_{n}, x\right)-\lambda_{k}^{2} F_{\nu}\left(\lambda_{n}, x\right) F_{\nu+1}\left(\lambda_{k}, x\right)\right\}\right]_{\delta}^{1} .
\end{aligned}
$$

It follows without difficulty from equations $(105 a)$ and (44) that at the upper limit the quantity in square brackets on the right hand side of (113) may be written

$$
\frac{\psi_{1}\left(\lambda_{n}\right)}{\lambda_{n}^{\nu-\frac{1}{2}}} .
$$

At the lower limit it may be written

if $\delta$ lies in the interval

$$
\frac{\psi_{1}\left(\lambda_{n}\right)}{\lambda_{n}^{\nu+\frac{1}{2}}},
$$

$$
0<\delta<\frac{c}{\lambda_{n}}
$$

* It is understood of course that the series on the right hand side of (112) is to be evaluated as a summable series, as we have yet no reason to suppose that it is convergent.

$\dagger$ This last reduction follows from the formula

$$
F_{\nu}^{\prime}(\lambda, x)=-\lambda^{2} F_{\nu+1}(\lambda, x)+\frac{\nu}{x} F_{\nu}(\lambda, x) .
$$

Cf. ByerLy, loc. cit., p. 223, formula (6). 
where $c$ is any positive constant $<1$, since we have from $(105 a)$

$$
F_{\nu}\left(\lambda_{n}, x\right)=\frac{J_{\nu}\left(\lambda_{n} x\right)}{\lambda_{n}^{\nu}}=\frac{\psi_{1}\left(\lambda_{n}\right)}{\lambda_{n}^{\nu}}, \quad F_{\nu+1}\left(\lambda_{n}, x\right)=\frac{\psi_{1}\left(\lambda_{n}\right)}{\lambda_{n}^{\nu+1}}
$$

If $\delta>c / \lambda_{n}$ it may be written

$$
\frac{\psi_{1}\left(\lambda_{n}\right)}{\lambda_{n}^{\nu-\frac{3}{2}}}
$$

since for such values of $\delta$ we have from $(105 a)$ and the asymptotic values of $J_{\nu}(x)$ and $J_{\nu+1}(x)$

$$
\begin{gathered}
F_{\nu}\left(\lambda_{n}, \delta\right)=\frac{\psi_{1}\left(\lambda_{n}\right)}{\sqrt{\lambda_{n} \delta \lambda_{n}^{\nu}}+\frac{\psi_{1}}{1}\left(\lambda_{n}\right)}\left(\lambda_{n} \delta\right)^{\frac{3}{2} \lambda_{n}^{\nu}}=\frac{1}{\sqrt{\delta}}\left[\frac{\psi_{1}\left(\lambda_{n}\right)}{\lambda_{n}^{\nu+\frac{1}{2}}}+\frac{\psi_{1}\left(\lambda_{n}\right)}{\left(\lambda_{n} \delta\right) \lambda_{n}^{\nu+\frac{1}{2}}}\right]=\frac{\psi_{1}\left(\lambda_{n}\right)}{\sqrt{\delta} \frac{\lambda_{n}^{\nu+\frac{1}{2}}}{F_{\nu+1}}}, \\
\left.F_{n}, \delta\right)=\frac{\psi_{1}\left(\lambda_{n}\right)}{\sqrt{\delta} \lambda_{n}^{\nu+\frac{3}{2}}} .
\end{gathered}
$$

Finally if $\delta=0$ the expression in square brackets on the right hand side of (113) vanishes at the lower limit. Hence we have

$$
\left|\int_{\delta}^{1} x F_{\nu}\left(\lambda_{n}, x\right) F_{\nu}\left(\lambda_{k}, x\right) d x\right|<\frac{M}{\lambda_{n}^{\nu-\frac{2}{2}} \cdot\left|\lambda_{n}^{2}-\lambda_{k}^{2}\right|} \quad(n \neq k, 0 \leqq \delta<1),
$$

where $M$ is a positive constant. Furthermore it follows from (46), (50), and $(105 a)$ that

$$
A_{n}=\frac{2 \lambda_{n}^{\nu+\frac{1}{2}}}{A} \int_{0}^{1} \sqrt{x} f(x) \cos \left(\lambda_{n} x-\alpha\right) d x+\lambda_{n}^{\nu-\frac{1}{2}} \psi_{1}\left(\lambda_{n}\right) .
$$

Consequently, in view of (114) and (115), we have for the general term of the series on the right hand side of (112)

$$
\begin{aligned}
& \left|A_{n} \int_{\delta}^{1} x F_{\nu}\left(\lambda_{n}, x\right) F_{\nu}\left(\lambda_{k}, x\right) d x\right| \\
& \quad<\frac{2 M \lambda_{n}}{A\left|\lambda_{n}^{2}-\lambda_{k}^{2}\right|}\left|\int_{0}^{1} \sqrt{x} f(x) \cos \left(\lambda_{n} x-\alpha\right) d x\right|+\frac{M\left|\psi_{1}\left(\lambda_{n}\right)\right|}{\left|\lambda_{n}^{2}-\lambda_{k}^{2}\right|} \quad(\delta \geqq 0) .
\end{aligned}
$$

The second term on the right hand side of the above inequality is, in view of the first inequality in (23), the general term of a convergent series, and it follows from Lemma 4 that the same is true for the first term. Hence the series on the right hand side of (112) converges uniformly for all values of $\delta$ in the interval $0 \leqq \delta<1$, and moreover it converges to the value of the integral on the left hand side for all values of $\delta>0$, since it is summable to that value. Since the convergence is uniform, we may let the $\delta$ of equation (112) approach zero. But in view of the fact that for the different values of $\lambda_{k}$ that enter, the functions $\sqrt{x} F_{\nu}\left(\lambda_{k}, x\right)$ are orthogonal to each other, all the terms but one on the right hand side of (112) drop out, and our lemma is established. 
Lemma 7. The function $\phi(x)$ associated with the series (106) does not become infinite to an order higher than $x^{-\frac{3}{2}}$ as we approach the point $x=0$.

By definition the function $\phi(x)$ is equal to the sum of two functions that are finite and integrable in the interval (43), plus the series

$$
\sum_{n=1}^{\infty} v_{n}(x)
$$

By Lemma 5 the series (116) is equal to the product of $x^{-\frac{3}{2}}$ into the series

$$
\sum_{n=1}^{\infty} \bar{v}_{n}(x) \text {. }
$$

But by the same lemma this latter series is uniformly summable and its terms are continuous in the interval (43). Hence it defines a continuous function in this interval, and consequently the series (116) defines a function that does not become infinite to an order higher than $x^{-\frac{3}{2}}$ when we approach the point $x=0$.

Our lemma is therefore proved.

THeOREM IV. If we have a function $\chi(x)$ which in the interval $0 \leqq x \leqq 1$ is finite everywhere save in the neighborhood of the point $x=0$, and if the integral

$$
\int_{0}^{1} x \chi(x) d x
$$

converges absolutely, and if furthermore

$$
\int_{0}^{1} x F_{\nu}\left(\lambda_{n}, x\right) \chi(x) d x=0 \quad(n=1,2,3, \cdots)
$$

where $\lambda_{1}, \lambda_{2}, \lambda_{3}, \cdots$, are the roots, positive or zero, of equation (107) arranged in increasing order of magnitude, then $\chi(x)$ is zero at every point at which it is continuous.

We know from some theorems due to DinI * that we can develop each of the functions

$$
x \sin k \pi x
$$

$$
(k=1,2,3, \cdots)
$$

in a series of the form

$$
a_{1} F_{\nu}\left(\lambda_{1}, x\right)+a_{2} F_{\nu}\left(\lambda_{2}, x\right)+a_{3} F_{\nu}\left(\lambda_{3}, x\right)+\cdots \dagger
$$

and that the series will converge to the value of the function developed at every point of the interval $0<x<1$. We are going to show further that for the functions we are considering the developments will converge uniformly throughout the interval $0 \leqq x \leqq 1$.

\footnotetext{
* Serie di Fourier, vol. 1. pp. 246-269. DINI treats a very general class of functions of which the functions (118) are a special case.

† There is an error in DiNi's formulæ for the special case in which $\lambda_{1}-0$; he omits the factor $F_{\nu}\left(\lambda_{1}, x\right)$ from the first term of the series.
} 
We will consider first the coefficient of the general term of the series (119) and for simplicity write $\lambda$ for $\lambda_{n}$. We have then

$$
a_{n}=\frac{\int_{0}^{1} x^{2} \sin k \pi x F_{\nu}(\lambda, x) d x}{\int_{0}^{1} x\left[F_{\nu}(\lambda, x)\right]^{2} d x} .
$$

It follows from equation (46) and equation (105a) that

$$
\int_{0}^{1} x\left[F_{\nu}(\lambda, x)\right]^{2} d x=\frac{A^{2}}{2 \lambda^{2 \nu+1}}+\frac{\psi_{1}(\lambda)}{\lambda^{2 \nu+2}} .
$$

We may therefore choose $\lambda$ so large that

But we have

$$
\left|a_{n}\right|<\frac{4 \lambda^{2 \nu+1}}{A^{2}}\left|\int_{0}^{1} x^{2} \sin k \pi x F_{\nu}(\lambda, x) d x\right|
$$

and if we set $\lambda x=y$

$$
\begin{aligned}
\int_{0}^{\frac{1}{\lambda}} x^{2} \sin k \pi x F_{\nu}(\lambda, x) d x & =\frac{1}{\lambda^{\nu}} \int_{0}^{\frac{1}{\lambda}} x^{2} \sin k \pi x J_{\nu}(\lambda x) d x \\
& =\frac{1}{\lambda^{\nu+3}} \int_{0}^{1} y^{2} \sin \frac{k \pi y}{\lambda} J_{\nu}(y) d y .
\end{aligned}
$$

Moreover we have from equations (49) and (105a)

$$
\begin{aligned}
& \int_{\frac{1}{\lambda}}^{1} x^{2} \sin k \pi x F_{v}(\lambda, x) d x \\
& =\frac{A}{\lambda^{\nu+\frac{1}{2}}} \int_{\frac{1}{\lambda}}^{1} x^{\frac{3}{2}} \sin k \pi x \cos (\lambda x-\alpha) d x+\frac{A^{\prime}}{\lambda^{\nu+\frac{\pi}{2}}} \int_{\frac{1}{\lambda}}^{1} x \sin k \pi x \sin (\lambda x-\alpha) d x+\frac{\psi_{1}(\lambda)}{\lambda^{\nu+\frac{\beta}{2}}} \\
& =\frac{A}{\lambda^{\nu+\frac{1}{2}}} \int_{0}^{1} x^{\frac{3}{2}} \sin k \pi x \cos (\lambda x-\alpha) d x+\frac{A^{\prime}}{\lambda^{\nu+\frac{3}{2}}} \int_{0}^{1} x^{\frac{1}{2}} \sin k \pi x \sin (\lambda x-\alpha) d x \\
& -\frac{A}{\lambda^{\nu+\frac{3}{2}}} \int_{0}^{\frac{1}{\lambda}} x^{\frac{3}{2}} \sin k \pi x \cos (\lambda x-\alpha) d x-\frac{A^{\prime}}{\lambda^{\nu+\frac{3}{2}}} \int_{0}^{\frac{1}{\lambda}} x^{\frac{1}{2}} \sin k \pi x \sin (\lambda x-\alpha) d x+\frac{\psi_{1}(\lambda)}{\lambda^{\nu+\frac{5}{2}}} .
\end{aligned}
$$

But we have from equation (57) for $\lambda=\lambda_{n}{ }^{*}$

$$
\cos (\lambda x-\alpha)=\cos \left(n \pi x+q x-\alpha+\frac{x \psi_{1}\left(\lambda_{n}\right)}{n}\right)
$$

* In the special case in which $\lambda_{1}=0$ we must take $\lambda=\lambda_{n+1}$ for (125) to hold true. However, since we are dealing here only with a question of convergence, this is unessential. 


$$
\begin{array}{r}
=\cos (n \pi x+q x-\alpha) \cos \frac{x \psi_{1}\left(\lambda_{n}\right)}{n}-\sin (n \pi x+q x-\alpha) \sin \frac{x \psi_{1}\left(\lambda_{n}\right)}{n} \\
=\cos (n \pi x+q x-\alpha)+[\cos (n \pi x+q x-\alpha)] \cdot\left[\cos \frac{x \psi_{1}\left(\lambda_{n}\right)}{n}-1\right] \\
-\sin (n \pi x+q x-\alpha) \sin \frac{x \psi_{1}\left(\lambda_{n}\right)}{n} .
\end{array}
$$

Moreover

$$
\begin{array}{r}
\int_{0}^{1} x^{\frac{3}{2}} \sin k \pi x \cos (n \pi x+q x-\alpha) d x=\int_{0}^{1} x^{\frac{3}{3}} \sin k \pi x \cos (q x-\alpha) \cos n \pi x d x \\
-\int_{0}^{1} x^{\frac{3}{2}} \sin k \pi x \sin (q x-\alpha) \sin n \pi x d x=\frac{\psi_{1}(n)}{n^{2}}, *
\end{array}
$$

and from an integration by parts

$$
\begin{aligned}
\int_{0}^{1} x^{\frac{3}{2}} \sin k \pi x \sin (n \pi x+q x-\alpha) \sin \frac{x \psi_{1}\left(\lambda_{n}\right)}{n} d x \\
=\frac{1}{n \pi+q}\left[-x^{\frac{3}{2}} \sin k \pi x \cos (n \pi x+q x-\alpha) \sin \frac{x \psi_{1}\left(\lambda_{n}\right)}{n}\right]_{0}^{1} \\
\quad+\frac{3}{2(n \pi+q)} \int_{0}^{1} x^{\frac{1}{2}} \sin k \pi x \cos (n \pi x+q x-\alpha) \sin \frac{x \psi_{1}\left(\lambda_{n}\right)}{n} d x \\
\quad+\frac{k \pi}{n \pi+q} \int_{0}^{1} x^{\frac{3}{2}} \cos k \pi x \cos (n \pi x+q x-\alpha) \sin \frac{x \psi_{1}\left(\lambda_{n}\right)}{n} d x \\
\quad+\frac{\psi_{1}\left(\lambda_{n}\right)}{n(n \pi+q)} \int_{0}^{1} x^{\frac{3}{2}} \sin k \pi x \cos (n \pi x+q x-\alpha) \cos \frac{x \psi_{1}\left(\lambda_{n}\right)}{n} d x
\end{aligned}
$$

Consequentiy, since

$$
\sin \frac{x \psi_{1}\left(\lambda_{n}\right)}{n}=\frac{\psi\left(x, \lambda_{n}\right)}{n}, \quad \cos \frac{x \psi_{1}\left(\lambda_{n}\right)}{n}-1=\frac{\psi\left(x, \lambda_{n}\right)}{n^{2}},
$$

it follows from (125), (126), and (127) that

$$
\int_{0}^{1} x^{\frac{3}{2}} \sin k \pi x \cos (\lambda x-\alpha) d x=\frac{\psi_{1}(n)}{n^{2}} .
$$

In a similar fashion it may be shown that

$$
\int_{0}^{1} x^{\frac{1}{2}} \sin k \pi x \sin (\lambda x-\alpha) d x=\frac{\psi_{1}(n)}{n},
$$

and consequently, since from Theorem I

$$
\lambda_{n}>C n,
$$

\footnotetext{
* Cf. Picard, Traité d'Analyse, 2d ed., vol. 1, p. 256.
} 
we have from (124), (128) and (129)

$$
\int_{\frac{1}{\lambda}}^{1} x^{2} \sin k \pi x F_{\nu}(\lambda, x) d x=\frac{\psi_{1}(n)}{n^{\nu+\frac{5}{2}}} .
$$

Combining (122), (123), (130), and (131) we obtain

$$
\int_{0}^{1} x^{2} \sin k \pi x F_{\nu}(\lambda, x) d x=\frac{\psi_{1}(n)}{n^{\nu+\frac{5}{2}}} .
$$

Since we know that the interval between two successive positive roots of equation (107) approaches $\pi$ as a limit, it follows without difficulty that a positive constant $M$ can be determined such that

$$
\lambda_{n}<M n \text {. }
$$

Combining (121), (132), and (133) we get

$$
\left|a_{n}\right|<\frac{K_{1} \lambda_{n}^{\nu}}{n^{\frac{3}{2}}}
$$

where $K_{1}$ is a positive constant. But since the Bessel function of any order has a maximum absolute value for all real positive values of the argument, we have from $(105 a)$

$$
\left|F_{\nu}\left(\lambda_{n}, x\right)\right|<\frac{K_{2}}{\lambda_{n}^{\nu}}
$$

where $K_{2}$ is a positive constant. Combining (134) and (135) we have

$$
\left|a_{n} F_{\nu}\left(\lambda_{n}, x\right)\right|<\frac{K}{n^{3}}
$$

$K$ being a positive constant. Hence the series that we are discussing converge absolutely and uniformly in the interval $0 \leqq x \leqq 1$.

Since the developments of the functions $(118)$ in series of the form (119) converge uniformly in the interval $0 \leqq x \leqq 1$, we have a right to multiply them by $x \chi(x)$ and integrate them term by term from 0 to 1 . Performing this operation we obtain in view of $(117)$

$$
\int_{0}^{1} x^{2} \sin k \pi x \chi(x) d x=0 \quad(k=1,2,3, \cdots) .
$$

Consequently, since any finite and integrable function whose Fourier's constants all vanish is zero at every point at which it is continuous, ${ }^{*}$ it follows that $x^{2} \chi(x)$ and hence $\chi(x)$ is zero at every point of the interval $0 \leqq x \leqq 1$ at which it is continuous.

*Cf. Hurwitz, Mathematisohe Annalen, vol. 57 (1903), p. 440. 
Theorem V. If $f(x)$ satisfies the conditions of Theorem II and if $\lambda_{1}, \lambda_{2}, \lambda_{3}, \ldots$ are the roots, positive or zero, of equation (107) arranged in order of increasing magnitude, then the series (106) will be summable to the value (110) at every point of the interval $0<x<1$ at which $f(x)$ is continuous or at which it has a finite jump, provided the latter points lie in an interval at every other point of which $f(x)$ is continuous; and will be uniformly summable to the value $f(x)$ in every closed interval lying in the interval $0<x<1$ which does not include a point of discontinuity of $f^{\prime}(x)$.

We have from equation (111)

$$
\int_{0}^{1} x[\phi(x)-f(x)] F_{\nu}\left(\lambda_{k}, x\right) d x=0 \quad(k=1,2,3, \cdots)
$$

where $\phi(x)$ is a function that has the value of the series (106) at every point at which $f(x)$ is continuous (see page 421).

By hypothesis $f(x)$ is finite and integrable in the interval $0 \leqq x \leqq 1$. We know from the definition of $\phi(x)$ that $\phi(x)$ is finite and integrable in the interval $0<\delta \leqq x \leqq 1$ where $\delta$ may be taken as small as we please. Moreover, it follows from Lemma 7 that $\left.\phi_{(}^{\prime} x\right)$ cannot become infinite at the point $x=0$ to an order higher than $x^{-\frac{3}{2}}$. Hence $\phi(x)$ is finite in the interval $0 \leqq x \leqq 1$ except in the neighborhood of the point $x=0$, and $x \phi(x)$ is absolutely integrable from 0 to 1 .

Consequently, in view of Theorem IV, it follows from equations (136) that

$$
\chi(x)=\phi(x)-f(x)=0
$$

at every point of the interval $0 \leqq x \leqq 1$ at which it is continuous. Now we know from the definition of $\phi(x)$ that $\phi(x)$ is continuous at every point of the interval $0<x<1$ at which $f(x)$ is continuous. Hence it follows from equation (137) that at every point of the interval $0<x<1$ at which $f(x)$ is continuous the series $(106)$ is summable to the value $(110) . *$

We consider next the points at which $f(x)$ has a finite jump and which lie in an interval at every other point of which $f(x)$ is continuous. We know that at every interior point of that interval, except the point at which $f(x)$ has a finite jump, the series (106) is summable to $f(x)$. Moreover, that series is the sum of a series that is uniformly summable and continuous in the neighborhood of

* Since $f(x)=\phi(x)$ at every point at which $f(x)$ is continuous and since from (109)

it follows that

$$
\phi(x)=f(x)+\sum_{n=1}^{\infty} w_{n}(x)
$$

$$
\sum_{n=1}^{\infty} w_{n}(x)
$$

is zero at every point at which $f(x)$ is continuons. However, since the series $(a)$ defines a function that is continuous throughout the interval $0<x \leqq 1$, it follows that it defines a function that is identically zero in that interval, so that $\phi(x) \equiv f(x)$. 
that point plus two series that are essentially Fourier developments of functions having a finite jump at that point. Hence the finite jump in the function defined by the sum of the two Fourier developments has the same magnitude as the finite jump in $f(x)$. However, it follows from FEJER's work that the Fourier developments are summable at a point at which the function they define has a finite jump, to a value half way between the limiting values of the function. Consequently the series $(106)$ is summable to the value (110) at the points which we are discussing.

That the series is uniformly summable to the value $f(x)$ in every closed interval lying in the interval $0<x<1$ which does not include a point of discontinuity of $f(x)$, is an immediate consequence of Theorem II and the fact that the series is summable to the value $f(x)$ at every point of such an interval.

\section{§4. Convergence factors and applications.}

Theorem VI. If the series

$$
u_{1}(x)+u_{2}(x)+u_{3}(x)+\cdots
$$

is uniformly summable in the interval $a \leqq x \leqq b$ and has the value $f(x)$, then the series

$$
u_{1}(x) e^{-\lambda_{1} a}+u_{2}(x) e^{-\lambda_{2} a}+u_{3}(x) e^{-\lambda_{3} a}+\cdots,
$$

where $\lambda_{1}, \lambda_{2}, \lambda_{3}, \ldots$ are the successive positive roots of (1), will be absolutely convergent in the region

$$
\left(\begin{array}{l}
a \\
a \leqq x \leqq b
\end{array}\right)
$$

and its value $u(\alpha, x)$ will approach $f(x)$ uniformly for all values of $x$ in the interval $a \leqq x \leqq b$, as a approaches +0 .

We will first show that the convergence factors

$$
e^{-\lambda_{1} a}, e^{-\lambda_{2} a}, e^{-\lambda_{3} a}, \cdots
$$

satisfy the conditions of a general theorem due to Bromwich.* It will then follow that the series (139) is absolutely convergent in the region (140) and that its value approaches $f(x)$ for all values of $x$ in the interval $a \leqq x \leqq b$ as $\alpha$ approaches +0 . That this approach is uniform can easily be seen by a slight modification of the reasoning in BROMwICH's theorem.

BROMwICH's conditions are

$$
\left.\begin{array}{c}
\sum_{n=\mu}^{n=\nu} n\left|\Delta^{2} v_{n}\right|<K \\
\lim _{n=\infty} n v_{n}=0
\end{array}\right\} \alpha>0,
$$

*Cf. Mathematische Annalen, vol. 65 (1908), p. 351. 
where $\mu$ and $\nu$ are any two integers, $v_{1}, v_{2}, v_{3}, \ldots$, are the convergence factors, $\Delta^{2} v_{n}$ represents their second differences, and $K$ is a positive constant. That the functions (141) satisfy $(\beta)$ and $(\gamma)$ is at once obvious. It only remains to examine $(\alpha)$.

In order to establish this latter condition we will prove that the second differences of the functions (141) satisfy the inequalities *

$$
\begin{array}{lr}
\left|\Delta^{2} e^{-\lambda_{n} a}\right|<N \alpha^{2}+\frac{M \alpha}{n^{2}} & (0<n a \leqq c ; n=1,2,3, \cdots), \\
\left|\Delta^{2} e^{-\lambda_{n} a}\right|<\frac{L}{n^{3} \alpha} & (a>0 ; n=1,2,3, \cdots) .
\end{array}
$$

It will then follow without difficulty that $(\alpha)$ holds. $\dagger$

Let

$$
\lambda_{n+1}-\lambda_{n}=\gamma_{n}, \quad \lambda_{n+2}-\lambda_{n+1}=\gamma_{n+1} .
$$

Then by Theorem I

and hence

$$
\left|\gamma_{n}-\pi\right|<\frac{K}{n^{2}}, \quad\left|\gamma_{n+1}-\pi\right|<\frac{K}{(n+1)^{2}}<\frac{K}{n^{2}},
$$

$$
\left|\gamma_{n+1}-\gamma_{n}\right|=\left|\left(\gamma_{n+1}-\pi\right)-\left(\gamma_{n}-\pi\right)\right| \leqq\left|\gamma_{n+1}-\pi\right|+\left|\gamma_{n}-\pi\right|<\frac{2 K}{n^{2}}
$$

But we have

$$
e^{-\lambda_{n} a}-2 e^{-\left(\lambda_{n}+\gamma_{n}\right) a}+e^{-\left(\lambda_{n}+2 \gamma_{n}\right) a}=e^{-\left(\lambda_{n}+\theta_{1} \gamma_{n}\right) a} \gamma_{n}^{2} \alpha^{2} \quad\left(0<\theta_{1}<2\right),
$$

and since, in view of (144), $\gamma_{n}$ has an upper limit, we obtain

$$
e^{-\lambda_{n} a}-2 e^{-\left(\lambda_{n}+\gamma_{n}\right) a}+e^{-\left(\lambda_{n}+2 \gamma_{n}\right) a}<N \alpha^{2},
$$

where $N$ is a positive constant.

We have furthermore from the Law of the Mean

$$
e^{-\lambda_{n+2} a}-e^{-\left(\lambda_{n}+2 \gamma_{n}\right) a}=-e^{-\left[\lambda_{n}+2 \gamma_{n}+\theta_{2}\left(\gamma_{n+1}-\gamma_{n}\right) a\right]}\left(\gamma_{n+1}-\gamma_{n}\right) \alpha \quad\left(\left|\theta_{2}\right|<1\right),
$$

from which we obtain with the aid of (145)

$$
\left|e^{-\lambda_{n+2 a}}-e^{-\left(\lambda_{n}+2 \gamma_{n}\right) \alpha}\right|<\frac{2 K \alpha}{n^{2}}=\frac{M \alpha}{n^{2}},
$$

where $M$ is a positive constant.

* It may be noted that the inequalities (142) and (143) are analogous to conditions $(d)$ and (e) in a theorem on convergence factors proved by the writer (these Transaotions, vol. 8 (1907), p. 300), which is a special case of BRomwICH's theorem. The necessity for distinguishing between the differences where $n a \leqq c$ and $n a>c$ often arises in the consideration of convergence factors that occur in practice.

† The proof of this fact in the present case is analogous to the proof in the case where conditions $(d)$ and $(e)$ of the theorem mentioned in the previous footnote hold. This latter proof is given on pp. 353 and 354 of Bromwich's article referred to above. 
Finally from (147) and (149) we have

$\left|e^{-\lambda_{n} a}-2 e^{-\lambda_{n+1} a}+e^{-\lambda_{n+2} a}\right| \leqq\left|e^{-\lambda_{n} a}-2 e^{-\left(\lambda_{n}+\gamma_{n}\right) a}+e^{-\left(\lambda_{n}+2 \gamma_{n}\right) a}\right|$

and hence the inequality (142) is satisfied.

$$
+\left|e^{-\lambda_{n+2 a}}-e^{-\left(\lambda_{n}+2 \gamma_{n}\right) a}\right|<N \alpha^{2}+\frac{M \alpha}{n^{2}},
$$

We obtain from (146), (148), and (145)

$$
\begin{aligned}
&\left|e^{-\lambda_{n} \alpha}-2 e^{-\lambda_{n+1} a}+e^{-\lambda_{n+2} \alpha}\right| \leqq e^{-\left(\lambda_{n}+\theta_{1} \gamma_{n}\right) a} \gamma_{n}^{2} \alpha^{2}+\left|e^{-\left[\lambda_{n}+2 \gamma_{n}+\theta_{2}\left(\gamma_{n+1}-\gamma_{n}\right)\right] a}\left(\gamma_{n+1}-\gamma_{n}\right) \alpha\right| \\
&<\frac{2 \gamma_{n}^{2} \alpha^{2}}{\left(\lambda_{n}+\theta_{1} \gamma_{n}\right)^{3} \alpha^{3}}+\frac{2 K \alpha}{n^{2}\left[\lambda_{n}+2 \gamma_{n}+\theta_{2}\left(\gamma_{n+1}-\gamma_{n}\right)\right]^{2} \alpha^{2}}<\frac{2 \gamma_{n}^{2}}{\lambda_{n}^{3} \alpha}+\frac{2 K}{n^{2} \lambda_{n}^{2} \alpha} \\
&<\frac{2 \gamma_{n}^{2}}{C^{3}}+\frac{2 K}{n^{3} \alpha}+\frac{C^{2}}{n^{4} \alpha}<\frac{L}{n^{3} \alpha} \quad(n=1,2,3, \cdots ; \alpha>0),
\end{aligned}
$$

where $L$ is a positive constant, and therefore the inequality (143) holds.

Consequently, as we have pointed out above, our theorem is proved.

An analogous theorem about slightly different convergence factors is the following:

Theorem VII. If the series (138) is uniformly summable in the interval $a \leqq x \leqq b$ and has the value $f(x)$, then the series

$$
u_{1}(x) e^{-\lambda_{1}^{2} a^{2}}+u_{2}(x) e^{-\lambda_{2}^{2} a^{2}}+u_{3}(x) e^{-\lambda_{3}^{2} a^{2}}+\cdots,
$$

where $\lambda_{1}, \lambda_{2}, \lambda_{3}, \ldots$ are the successive positive roots of $(1)$, will be absolutely convergent in the region (140) and its value $u(\alpha, x)$ will approach $f(x)$ uniformly for all values of $x$ in the interval $a \leqq x \leqq b$, as $\alpha$ approaches +0 .

This theorem also can be proved by showing that the convergence factors

$$
e^{-\lambda_{1}^{2} a^{2}}, e^{-\lambda_{2}^{2} a^{2}}, e^{-\lambda_{3}^{2} a^{2}}, \cdots
$$

satisfy the conditions of Bromwich's theorem. The proof is quite analogous to the previous proof, save that instead of the inequalities (142) and (143) we establish the slightly different inequalities

$$
\begin{aligned}
& \left|\Delta^{2} e^{-\lambda_{n}^{2} a^{2}}\right|<N \alpha^{2} \quad(0<n a \leqq c ; n=1,2,3, \cdots), \\
& \left|\Delta^{2} e^{-\lambda_{n}^{2} a^{2}}\right|<\frac{L}{n^{4} \alpha^{2}} \quad(\alpha>0 ; n=1,2,3, \cdots) .
\end{aligned}
$$

The results just obtained have a direct application to many problems in mathematical physics.

Problem $I$. The problem of determining the flow of heat in an infinite, circular cylinder, when the initial and boundary conditions depend only on the 
perpendicular distance from the axis of the cylinder, reduces to the determination of a continuous function * of $r$ and $z, v(r, z)$ such that

in the region

$$
\frac{\partial v}{\partial z}=a\left(\frac{\partial^{2} v}{\partial r^{2}}+\frac{1}{r} \frac{\partial v}{\partial r}\right)
$$

and

$$
\left(\begin{array}{c}
z>0 \\
0<r<1
\end{array}\right)
$$

$$
\begin{array}{cr}
l \frac{\partial v}{\partial r}+h v=0 & (z>0, r=1), \\
v=f(r) & (z=0),
\end{array}
$$

where $a, l$, and $h$ are constants and $f(r)$ is a function of $r$ that gives the initial distribution of the temperature. $\dagger$

It can be shown easily that the function

$$
A e^{-a \lambda^{2} z} F_{0}(\lambda, r)
$$

where $A$ is a constant and $\lambda$ is a root of the equation

$$
l \lambda J_{0}^{\prime}(\lambda)+h J_{0}(\lambda)=0
$$

satisfies conditions (154) and (156). It remains to construct from functions of the form (158) a continuous function which will also satisfy (154) and (156) and in addition will approach continuously the boundary value $f(r)$, where $f(r)$ is continuous, and which will remain finite where that is not the case.

Consider the series

$$
A_{1} e^{-a \lambda_{1}^{2} z} F_{0}\left(\lambda_{1}, r\right)+A_{2} e^{-a \lambda_{2}^{2} z} F_{0}\left(\lambda_{2}, r\right)+\cdots
$$

where the $\lambda$ 's are the roots, positive or zero, of equation (107) for $\nu=0$ arranged in order of increasing magnitude and the $A$ 's are the coefficients of the series of the form (106) corresponding to the given function $f(r)$. If the function $f(r)$ is finite and integrable in the interval $0 \leqq r \leqq 1$, we obtain from $(46),(50)$, and $(105 a)$

$$
\left|A_{n} F_{0}\left(\lambda_{n}, r\right)\right|<K \sqrt{\lambda_{n}}
$$$$
(0 \leqq r \leqq 1)
$$

where $K$ is a positive constant. Hence for the general term of $(160)$ we have in view of the first inequality in (23)

\footnotetext{
* By a continuous function we mean that $v(r, z)$ is to be continuous in the two variables $r$ and $z$ throughout the region

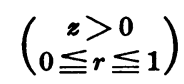

and is to approach the boundary value $f(r)$ continuously wherever $f(r)$ is continuous.

†Cf. CarsLaw, Introduction to the Theory of Fourier's Series and Integrals, etc., Chapter XV.
} 


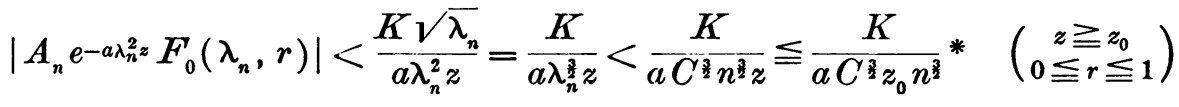

and therefore the series (160) is uniformly convergent in the region

$$
\left(\begin{array}{l}
0 \leqq r \leqq 1 \\
z \geqq z_{0}>0
\end{array}\right)
$$

Since, moreover, the terms of (160) are continuous functions of $r$ and $z$ for all values of $r$ and $z$, it follows that the series defines a continuous function of $r$ and $z$ throughout the region (161), and therefore, since $z_{0}$ is any constant greater than zero, throughout the region

$$
\left(\begin{array}{c}
0 \leqq r \leqq 1 \\
z>0
\end{array}\right)
$$

In similar fashion it may be shown that the function defined by $(160)$ satisfies conditions (154) and (156). It only remains to consider the behavior of the function as we approach the line $z=0$ from the positive side.

We know from Theorem $\mathrm{V}$ that if $f(r)$ is a function that is finite and integrable in the interval $0 \leqq r \leqq 1$, then the series

$$
A_{1} F_{0}\left(\lambda_{1}, r\right)+A_{2} F_{0}\left(\lambda_{2}, r\right)+\cdots,
$$

where the $A$ 's and $\lambda$ 's are those of the series $(160)$, will be uniformly summable to the value of $f(r)$ throughout every interval that lies within a larger interval in which $f(r)$ is continuous. It follows then from Theorem VII $\dagger$ that, as $z$ approaches zero through positive values, the function defined by $(160)$ will approach the value $f(r)$ uniformly throughout any $r$-interval lying in a larger interval in which $f(r)$ is continuous. Consequently, if $r^{\prime}$ is any interior point of an interval in which $f(r)$ is continuous, the function defined by (160) approaches the value $f\left(r^{\prime}\right)$ as we approach the point $\left(r=r^{\prime}, z=0\right)$ along any path lying in the region (155).

We now wish to examine the behavior of the function defined by $(160)$ when we approach a point that is not an interior point of an interval of continuity of $f(r)$. If we define $S_{n}(r)$ for the series (163) in the same way that $S_{n}(x)$ was defined in (34) for a corresponding series, we may, for values of $z>0$, write the series $(160)$ in the form $\ddagger$

$$
\sum_{n=1}^{\infty} n S_{n}(r)\left[e^{-a \lambda_{n}^{2 z}}-2 e^{-a \lambda_{n+1}^{2} z}+e^{-a \lambda_{n+2}^{2} z}\right] .
$$

${ }^{*}$ If $\lambda_{1}=0$ we must take the $(n+1)$ st term as the general term instead of the $n$th term in order that this inequality may hold.

$\dagger$ In this theorem we did not consider the possibility of $\lambda_{1}$ being equal to zero. However, this is an unessential point, as the behavior of a finite number of convergence factors at the beginning of the series does not matter, provided they approach 1 as the parameter of which they are functions approaches zero.

†Cf. equation (7) of the writer's article in these Transactions referred to on page 430. 
If now we make the change of variable

the expression (164) reduces to

$$
a z=\alpha^{2},
$$

$$
\sum_{n=1}^{\infty} n S_{n}(r)\left[e^{-\lambda_{n}^{2} a^{2}}-2 e^{-\lambda_{n+1}^{2} a^{2}}+e^{-\lambda_{n+2}^{2} a^{2}}\right] .
$$

But we have from the corollary of Theorem II*

$$
\left|S_{n}(r)\right|<M \quad\left(n=1,2,3, \cdots ; 0<r_{0} \leqq r<1\right),
$$

where $M$ is a positive constant. Consequently, since the convergence factors (151) satisfy the conditions of BROMwICH's theorem, the series (165) is, throughout the region

less in absolute value than

$$
\left(\begin{array}{c}
a>0 \\
0<r_{0} \leqq r \leqq 1
\end{array}\right),
$$

$$
M \sum_{n=1}^{\infty} n\left|e^{-\lambda_{n}^{2} a^{2}}-2 e^{-\lambda_{n+1}^{2} a^{2}}+e^{-\lambda_{n+2}^{2} a^{2}}\right|<M K,
$$

where $K$ is a positive constant. Hence if we approach any point $\left(r=r^{\prime}, t=0\right)$ such that

$$
0<r_{0}<c \leqq r^{\prime} \leqq 1,
$$

along any path lying in the region (155), the function defined by (160) remains finite.

Problem II. The problem of determining the steady flow of heat in a semiinfinite circular cylinder whose base is kept at a temperature

$$
v=f(r),
$$

where $r$ is the perpendicular distance from the axis of the cylinder, reduces to the determination of a continuous function $\dagger$ of $r$ and $z$ which satisfies

$$
\frac{1}{r} \frac{\partial}{\partial r}\left(r \frac{\partial v}{\partial r}\right)+\frac{\partial^{2} v}{\partial z^{2}}=0
$$

in the region (155), and also satisfies (156) and (157).

It can be shown easily that the function

$$
A e^{-\lambda z} F_{0}(\lambda, r) \text {, }
$$

where $A$ is a constant and $\lambda$ is a root of equation (159), satisfies (166) and (156). We form now the series

* This corollary was proved with regard to the series (35), but as (35) differs from (106) by at most one term that is finite for all values of the variable involved, it holds for the latter series also.

†Cf. note, p. 432.

$\ddagger$ Cf. Carslaw, loc. cit. : p. 321 


$$
A_{1} e^{-\lambda_{1} z} F_{0}\left(\lambda_{1}, r\right)+A_{2} e^{-\lambda_{2} z} F_{0}\left(\lambda_{2}, r\right)+\cdots,
$$

where the $\lambda$ 's are the roots, positive or zero, of equation (107) for $\nu=0$ arranged in increasing order of magnitude, and the $A$ 's are the coefficients of the series of the form (106) corresponding to the given function $f(r)$.

If $f(r)$ is finite and integrable in the interval $0 \leqq r \leqq 1$, the series (167) will converge for $z>0$ to a continuous function of $r$ and $z$ that satisfies (166) and (156), and approaches the proper boundary value when we approach a point on the line $z=0$ that lies within an interval of continuity of $f(r)$. Furthermore the series (167) remains finite when we approach any point on the line $z=0$ that lies in the interval

$$
0<c \leqq r \leqq 1 \text {. }
$$

The proof of the above facts is analogous to the proof in the previous proklem, except that we use Theorem VI instead of Theorem VII.

The University of Cincinnati, Cincinnati, Ohio. 